\title{
Optical configuration and analysis of the AMBER/VLTI instrument
}

\author{
S. Robbe-Dubois ${ }^{1}$, S. Lagarde ${ }^{2}$, R. G. Petrov ${ }^{1}$, F. Lisi ${ }^{3}$, U. Beckmann ${ }^{4}$, P. Antonelli ${ }^{2}$, Y. Bresson ${ }^{2}$, \\ G. Martinot-Lagarde ${ }^{2,7}$, A. Roussel ${ }^{2}$, P. Salinari ${ }^{3}$, M. Vannier 1,6,13, A. Chelli ${ }^{5}$, M. Dugué2 ${ }^{2}$ G. Duvert ${ }^{5}$, S. Gennari ${ }^{3}$, \\ L. Glück ${ }^{5}$, P. Kern ${ }^{5}$, E. Le Coarer ${ }^{5}$, F. Malbet ${ }^{5}$, F. Millour ${ }^{1,5}$, K. Perraut ${ }^{5}$, P. Puget ${ }^{5}$, F. Rantakyrö ${ }^{6}$, E. Tatulli ${ }^{5}$, \\ G. Weigelt ${ }^{4}$, G. Zins ${ }^{5}$, M. Accardo ${ }^{3}$, B. Acke ${ }^{5,14}$, K. Agabi ${ }^{1}$, E. Altariba ${ }^{5}$, B. Arezki ${ }^{5}$, E. Aristidi ${ }^{1}$, C. Baffa ${ }^{3}$,
} J. Behrend ${ }^{4}$, T. Blöcker ${ }^{4}$, S. Bonhomme ${ }^{2}$, S. Busoni ${ }^{3}$, F. Cassaing ${ }^{8}$, J.-M. Clausse ${ }^{2}$, J. Colin ${ }^{2}$, C. Connot ${ }^{4}$, L. Delage ${ }^{10}$, A. Delboulbé ${ }^{5}$, A. Domiciano de Souza ${ }^{1,2}$, T. Driebe ${ }^{4}$, P. Feautrier $^{5}$, D. Ferruzzi ${ }^{3}$, T. Forveille ${ }^{5}$, E. Fossat $^{1}$, R. Foy ${ }^{9}$, D. Fraix-Burnet ${ }^{5}$, A. Gallardo ${ }^{5}$, E. Giani ${ }^{3}$, C. Gil ${ }^{5,15}$, A. Glentzlin ${ }^{2}$, M. Heiden ${ }^{4}$, M. Heininger ${ }^{4}$, O. Hernandez Utrera ${ }^{5}$, K.-H. Hofmann ${ }^{4}$, D. Kamm² ${ }^{2}$, M. Kiekebusch ${ }^{6}$, S. Kraus ${ }^{4}$, D. Le Contel ${ }^{2}$, J.-M. Le Contel ${ }^{2}$, T. Lesourd ${ }^{7}$, B. Lopez ${ }^{2}$,

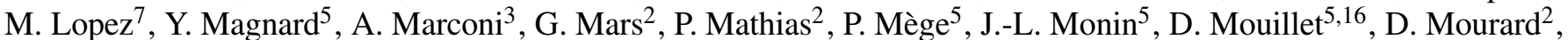
E. Nussbaum ${ }^{4}$, K. Ohnaka ${ }^{4}$, J. Pacheco ${ }^{2}$, C. Perrier ${ }^{5}$, Y. Rabbia ${ }^{2}$, S. Rebattu ${ }^{2}$, F. Reynaud ${ }^{10}$, A. Richichi ${ }^{11}$, A. Robini ${ }^{1}$, M. Sacchettini ${ }^{5}$, D. Schertl ${ }^{4}$, M. Schöller ${ }^{6}$, W. Solscheid ${ }^{4}$, A. Spang ${ }^{2}$, P. Stee ${ }^{2}$, P. Stefanini ${ }^{3}$, M. Tallon ${ }^{9}$, I. Tallon-Bosc ${ }^{9}$, D. Tasso ${ }^{2}$, L. Testi ${ }^{3}$, F. Vakili ${ }^{1}$, O. von der Lühe ${ }^{12}$, J.-C. Valtier ${ }^{2}$, and N. Ventura ${ }^{5}$

(Affiliations can be found after the references)

Received 13 April 2006 / Accepted 28 August 2006

\section{ABSTRACT}

\begin{abstract}
Aims. This paper describes the design goals and engineering efforts that led to the realization of AMBER (Astronomical Multi BEam combineR) and to the achievement of its present performance.

Methods. On the basis of the general instrumental concept, AMBER was decomposed into modules whose functions and detailed characteristics are given. Emphasis is put on the spatial filtering system, a key element of the instrument. We established a budget for transmission and contrast degradation through the different modules, and made the detailed optical design. The latter confirmed the overall performance of the instrument and defined the exact implementation of the AMBER optics.

Results. The performance was assessed with laboratory measurements and commissionings at the VLTI, in terms of spectral coverage and resolution, instrumental contrast higher than 0.80 , minimum magnitude of 11 in $K$, absolute visibility accuracy of $1 \%$, and differential phase stability of $10^{-3} \mathrm{rad}$ over one minute.
\end{abstract}

Key words. instrumentation: high angular resolution - instrumentation: interferometers - methods: analytical - methods: numerical methods: laboratory - techniques: high angular resolution

\section{Introduction}

AMBER (Petrov et al. 2007) is the near infrared focal beam combiner of the Very Large Telescope Interferometric mode (VLTI). A consortium of French, German, and Italian institutes is in charge of the specification, design, construction, integration, and commissioning of AMBER (http://amber.obs.ujf-grenoble.fr). This instrument is designed to combine three beams coming from any combination of Unit 8-m Telescopes (UT) or Auxiliary 1.8-m Telescopes (AT). The spectral coverage is from 1 to $2.4 \mu \mathrm{m}$ with a priority to the $K$ band.

AMBER is a general user instrument with a very wide range of astrophysical applications, as is shown in Richichi et al. (2000) and Petrov et al. (2007). To achieve the ambitious programs, Petrov et al. (2001) presented the associated specifications and goals, such as:

- Spectral coverage: $J, H$, and $K$ bands, from $1.0 \mu \mathrm{m}$ to $2.3 \mu \mathrm{m}$ (goal: up to $2.4 \mu \mathrm{m}$ ).

- Spectral resolutions: minimum spectral resolution $30<R<$ 50 , medium spectral resolution $500<R<1000$, and highest spectral resolution $10000<R<15000$.
- Minimum magnitude: $K=11, H=11$ (goals: $K=13$, $H=12.5, J=11.5)$.

- Absolute visibility accuracy: $3 \sigma_{V}=0.01$ (goal: $\sigma_{V}=10^{-4}$ ).

- Differential phase stability: $10^{-3} \mathrm{rad}$ (goal: $10^{-4} \mathrm{rad}$ ) over one minute integration. This allows us to compute phase closure that is necessary in the search of brown dwarfs and extra solar planets (Segransan et al. 2000).

These specifications have been the starting point of a global system analysis (Malbet et al. 2003) initiated by a group of interferometrists from several French institutes and completed by the Interferometric GRoup (IGR) of AMBER. This work led to the current definition of the AMBER instrument whose broad outlines are recalled in the paragraphs below.

To reach a sufficient sensitivity, ESO provided a 60 actuator curvature sensing system MACAO (Multi-Application Curvature Adaptive Optics) (Arsenault et al. 2003) specified to deliver at least a 50\% Strehl ratio @ $2.2 \mu \mathrm{m}$ for on-axis bright sources $(V=8)$ under median seeing conditions $\left(0.65^{\prime \prime}\right)$ and a 25\% Strehl ratio@2.2 $\mu \mathrm{m}$ for faint sources $(V=15.5)$ under the same seeing conditions. The required high accuracy of the absolute visibility measurements implies the use of spatial filters (Mège et al. 2000; Tatulli et al. 2004) with single mode 
fibers based on the experience of other smaller, successful interferometers such as IOTA/FLUOR (Coudé du Foresto 1997). The atmospheric noise is reduced to photometric fluctuations, which can be monitored, and to Optical Path Difference (OPD) fluctuations between the different pupils, which can be frozen by very short exposures or adaptively corrected by a fringe tracker.

The simultaneous observation of different spectral channels is ensured by dispersed fringes. This very significantly increases the number and the quality of the measurements and subsequently the constraints imposed on the atmospherical models. The modularity of the concept was a strong argument in favor of the multi-axial scheme, as carried out on the Grand Interferomètre à 2 Télescopes (GI2T) of the Plateau de Calern (Mourard et al. 2000). In addition, it was demonstrated that the instrument must correct the atmospheric transversal dispersion in $J$ and $H$ (Tallon-Bosc 1999). The need of an image cold stop was assessed by Malbet (1999) to reduce the thermal background coming from the blackbody emission of the fiber heads, which can be greater than the detector RON, especially for long time exposures in the $K$-Band. A pupil mask also acts as a cold stop. To perform the data reduction, the ABCD algorithm (Chelli 2000; Millour et al. 2004), as used with co-axial configurations with a temporal coding, was chosen. The associated complete data calibration procedure was then fully defined (Hofman 1999; Chelli 2000; Tatulli et al. 2007).

On the basis of the general instrumental concept resulting from the global system analysis, we defined the main modules and necessary accessories (such as the alignment units) of AMBER. We established a budget for throughput and contrast degradation through the different modules, made the detailed optical design, and performed a complete optical analysis. The latter confirmed the expected overall performance of the instrument in terms of signal-to-noise ratio (SNR). This entire process is described in the present paper. The procedure described here to allocate the specifications of the different modules of an interferometer could be used, after some changes, for the design of other interferometrical instruments, such as, for example, the VLTI second generation instruments.

\section{Overview of the AMBER implementation}

The concept of AMBER is illustrated by Fig. 1. Each input beam is fed into a single mode fiber that reduces all chromatic wavefront perturbations to photometric and global OPD fluctuations (6). At the output of the fibers, the beams are collimated, maintained parallel, and then focused in a common Airy disk (1). The latter contains Young fringes with spacings specific to each baseline, allowing us to separate the interferograms in the Fourier space. This Airy disk goes through the spectrograph slit (3) after being anamorphosed by cylindrical optics (2). The spectrograph (4) forms dispersed fringes on the detector (5), where each column allows us to analyse the interferograms in a different spectral channel. A fraction of each beam is collected before the beam combination to monitor the photometry variations (7).

Figure 2 shows the global implementation of AMBER, and Fig. 3 shows a picture of the instrument taken at the end of the integration at Paranal (March 2004). The core of the instrument is composed of the following modules, filling specific functions:

- SPatial Filters (SPF) to spatially filter the wavefront perturbation and reach high-visibility precision measurements. The functions of this element are also: spectral band selection $(J, H$, and $K)$, interferometric arm selection, control of

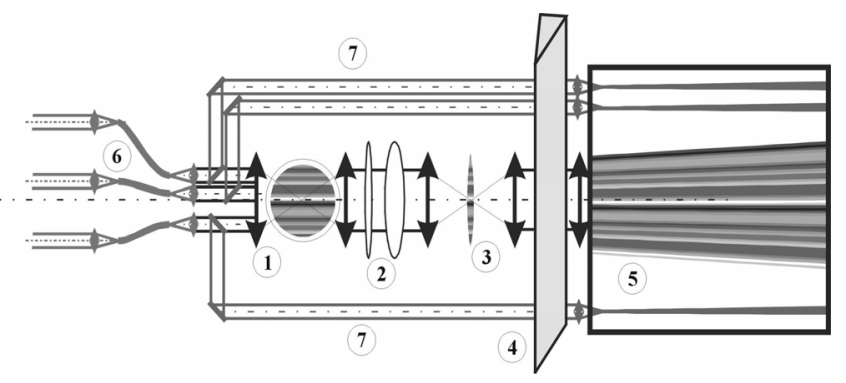

Fig. 1. Scheme of the AMBER configuration: (1) multiaxial beam combiner; (2) cylindrical optics; (3) anamorphosed focal image with fringes; (4) "long slit spectrograph"; (5) dispersed fringes on 2D detector; (6) spatial filter with single mode optical fibers; (7) photometric beams.

the beam size and position, flux optimization, OPD equalization, polarization control, and combination of the spectral bands.

- ANamorphosis System (ANS) to compress the beams in one direction without perturbing the pupil location.

- Cooled SPectroGraph (SPG). This element includes: dispersion with different resolutions, thermal noise reduction, pupil configuration, photometric calibration, and spectral filtering.

- Cooled DETector (DET), which detects the dispersed fringes.

The auxiliary modules are:

- System to correct the atmospheric transversal dispersion (ADC) in $J$ and $H$.

- Calibration and Alignment Unit (CAU) necessary to perform the contrast calibration (Millour et al. 2004; Tatulli et al. 2007).

- Remote Artificial Sources (RAS) allowing for the alignments, the spectral calibration, and feeding the CAU for the contrast calibration.

- Matrix Calibration System (MCS) scheduled to calibrate the contrast to achieve specific scientific goals.

- A BYPass (BYP) of the SPF to align the warm instrument in the visible (for controlling the pupil, the image location, and the beam separation and height), and to inject light directly to SPG.

We will describe each module in detail in the following paragraphs, from the entrance of AMBER to the detector, starting with the main modules and continuing with the auxiliary ones.

\section{The spatial filters}

The three VLTI beams at the entrance of AMBER have a diameter of $18 \mathrm{~mm}$ and equal separations of $240 \mathrm{~mm}$ (see Fig. 2). The three AMBER beams are separated by $70 \mathrm{~mm}$ at the fiber entrance. The separations at the entrance are achieved by adjusting the VLTI beam injection optics, allowing us to compensate for the optical path difference with additional path lengths. The chosen configuration ensures perfect symmetry between the interferometric paths and allows for the use of small size optics.

\subsection{Characteristics of the spatial filtering}

Single mode fibers cannot be efficient over a too large wavelength domain. The full $J, H, K$ range from 1 to $2.4 \mu$ m needs at least two different fibers. The most efficient way is to use one spatial filter by spectral band, avoiding dividing the $H$-band in 


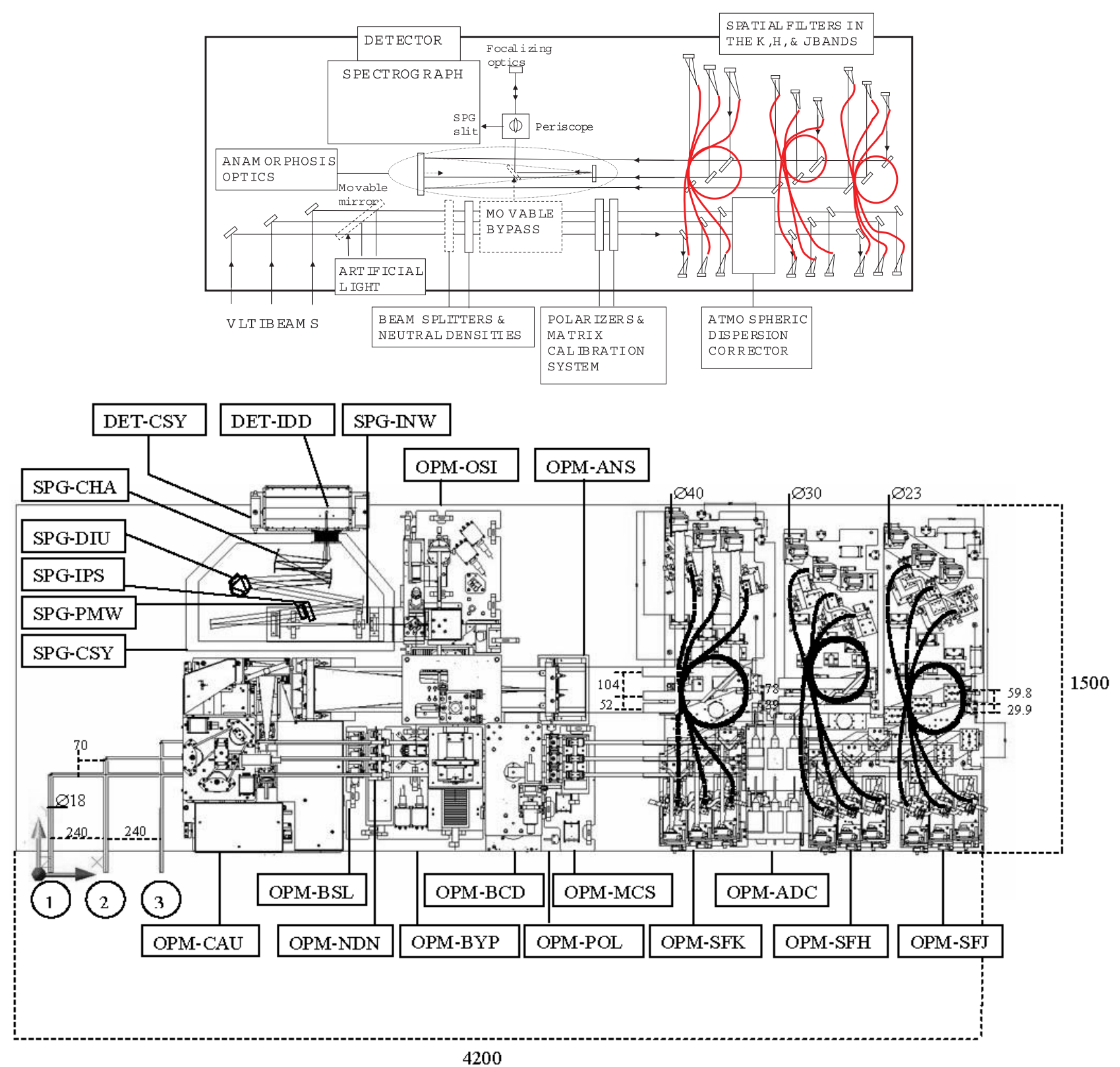

Fig. 2. General implementation of AMBER. The top scheme shows the light path from the VLTI to the detector. Detailed configuration below. The VLTI beams arrive in the lower left corner. OPM-CAU: Calibration and Alignment Unit. OPM-BCD: beam inverting device (Petrov et al. 2003). OPM-POL: polarization selecting device. OPM-SFK (SFH, SFJ): spatial filters for the $K$-, $H$-, and $J$-bands. OPM-ADC: corrector for the atmospheric differential refraction in $H$ and $J$. OPM-ANS: cylindrical afocal system for image anamorphosis. OPM-OSI: periscope to co-align the warm and the cold optics. OPM-BYP: movable bypass directly sending the VLTI beams towards technical tools or towards the spectrograph to check VLTI alignment and acquire complex fields. SPG-INW: input wheel with image cold stop and diaphgram inside the spectrograph (SPG). SPG-PMW: pupil mask wheel. SPG-IPS: beam splitter allowing the separation between interferometric and photometric beams. SPG-DIU: light dispersion (gratings or prism). SPG-CHA: SPG camera. DET-IDD: chip. SPG-CSY and DET-CSY: cryostats of the SPG and of the Hawaii detector (DET). During final operation, the two cryostats are connected by a cold tunnel and share the same vacuum.

two, which would result in the loss of a part of the $H$-band. The spatial filtering modules $\mathrm{SFJ}(H, K)$ (Fig. 4) receive the light from dichroics. Parabolic mirrors inject the light in silicate birefringent single-mode fibers. At the exit of the fibers the same optical system is repeated.

\subsubsection{The optical fibers}

LAOG provided equalized $J$-fibers, $H$-fibers, and $K$-fibers with a maximal accuracy of $\pm 20 \mu \mathrm{m}$. The characteristics of the presently used fibers are given in Table 1.
The specifications on spatial filtering are driven by the quality of the optical fibers. The fiber length of about $1.30 \mathrm{~m}$ ensures a good transmission while maintaining a $10^{-3}$ attenuation of the high order propagation modes (Malbet et al. 2003). The polarization-maintaining is achieved by fibers with an elliptical core causing the so-called "form birefringence", or by strong birefringence caused by two stress members applied on opposite sides of the core (bow tie- and panda-type). The fibers create an intensity modulation in some of the images recorded by AMBER. Figure 5 shows this modulation for optical fibers used previously to those of Table 1 . This effect, fainter now, is present 


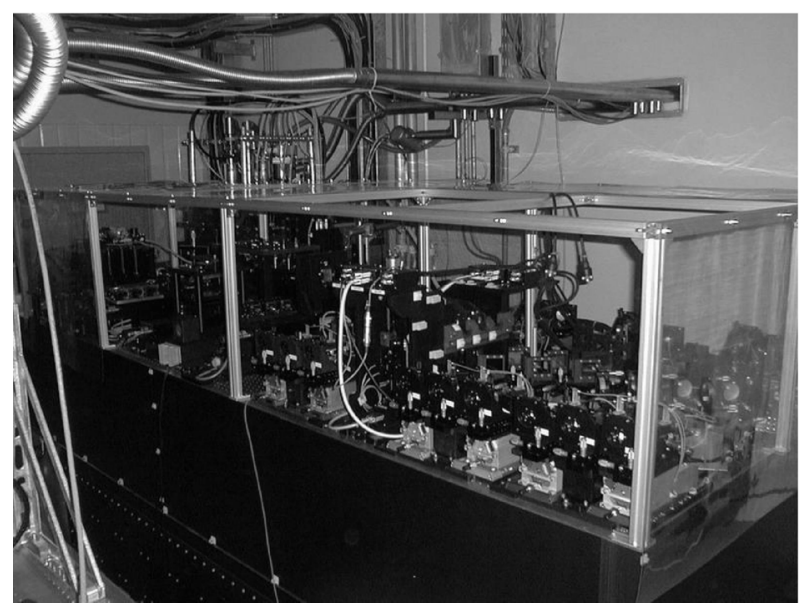

Fig. 3. Picture of the AMBER instrument at the end of the integration at Paranal in March 2004 (by A. Delboulbé, LAOG).
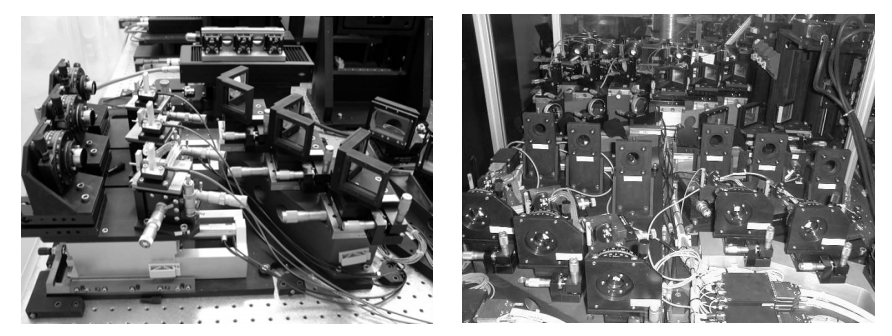

Fig. 4. Pictures of the $K$-spatial filter entrance. Left (picture by Y. Bresson, OCA): the three beams meet the dichroics and the parabolic off-axis mirrors before the injection through the Si birefringent singlemode fibers; Right (picture by A. Delboulbé, LAOG): the optical configuration is repeated at the exit of the fibers. Diaphragms control the beam size at the exit of the fibers and shutters select the interferometric arms.

Table 1. Manufacturer and characteristics of the fibers in the $K-, H$-, and $J$-bands. NA: numerical aperture, $\lambda_{\mathrm{c}}$ : cut-off wavelength, $\oslash$ : diameters, Conc.: core concentricity.

\begin{tabular}{lll}
\hline \hline$K$-band & Highwave & NA $=0.16 ; \lambda_{\mathrm{c}}=1900 \mathrm{~nm}$ \\
Silica & Core $\oslash=9.7 \mu \mathrm{m} ;$ Conc. $<5 \mu \mathrm{m}$ \\
Elliptical core & Coating $\oslash=245 \mu \mathrm{m}$ \\
& Absolute length $=1.30 \mathrm{~m} \pm 0.01 \mathrm{~m}$ \\
& Length difference after polishing: $\leq 11 \mu \mathrm{m}$ \\
\hline$H$-band & Fujikura & NA $=0.15 ; \lambda_{\mathrm{c}}=1150 \mathrm{~nm}$ \\
Silica & Mode $\oslash=5.5 \mu \mathrm{m} @ 1300 \mathrm{~nm}$; Conc. $0.2 \mu \mathrm{m}$ \\
Panda core & Coating $\oslash=245 \mu \mathrm{m}$ \\
& Absolute length $=1.30 \mathrm{~m} \pm 0.01 \mathrm{~m}$ \\
& Length difference after polishing: $\leq 12 \mu \mathrm{m}$ \\
\hline$J$-band & Fibercore & NA $=0.14 ; \lambda_{\mathrm{c}}=944 \mathrm{~nm}$ \\
Silica & Mode $\oslash=6.3 \mu \mathrm{m} @ 1060 \mathrm{~nm} ;$ Conc. $0.28 \mu \mathrm{m}$ \\
Bow tie core & Coating $\oslash=245 \mu \mathrm{m}$ \\
& Absolute length $=1.30 \mathrm{~m} \pm 0.01 \mathrm{~m}$ \\
& Length difference after polishing: $\leq 20 \mu \mathrm{m}$ \\
\hline
\end{tabular}

in the science as well as in the calibrator source and can then be reduced below the specifications in the calibration process. However, it depends on the fiber temperature and on the injection conditions (conditioned by the Strehl ratio). As far as the highest accuracy goals are concerned (especially that of reaching very high accuracy differential phase measurements), a fast correct calibration of this effect is necessary.

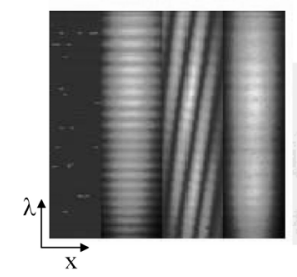

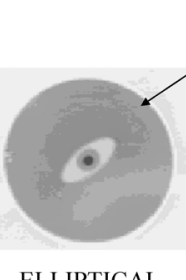

ELLIPTICAL

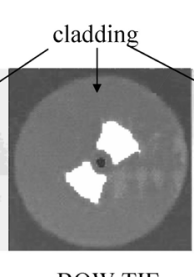

BOW TIE zones of

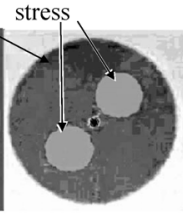

PANDA
Fig. 5. Left: illustration of photometric images (extreme) and interferometric image (center) produced with the artificial source ( $H$-band, $\Delta \lambda=150 \mathrm{~nm})$. The zero OPD is not exact. Note the intensity modulation generated by the fibers. Right: cross-sections of polarization maintaining fibres: elliptical core, bow tie, and panda fibers (http://www.highwave-tech.com/; http://www. fibercore. com/).

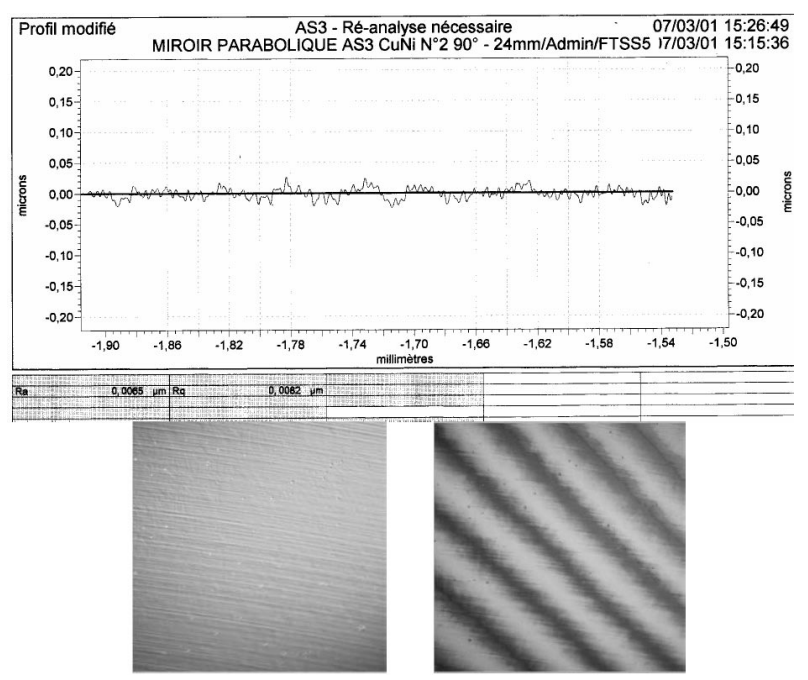

Fig. 6. Above is the surface profile as measured with the micro-sensor of SAVIMEX on a test element: maximum roughness of $60 \mathrm{~nm}$ PTV and rms roughness of Ra $6.5 \mathrm{~nm}$ and $\mathrm{Rq} 8.2 \mathrm{~nm}$; Below is the picture of the mirror allowing the manufacturing of the three injection optics in the SFJ as observed through the microscope (field of $400 \times 300 \mu \mathrm{m}$ ). The PTV roughness is about $1 / 4$ th to $1 / 8$ th of fringe, i.e., 80 to $40 \mathrm{~nm}$. The rms roughness is about $6 \mathrm{~nm}$. The surface optical quality measured with the collimating lunette is below $30 \mathrm{~nm}$, i.e., $\lambda / 20$ PTV @ $633 \mathrm{~nm}$ on an 18 -mm diameter disk.

\subsubsection{Fiber injection}

The parabolic mirrors, designed by the French company SAVIMEX (Grasse), are metallic off-axis diamond-turned mirrors. They were controlled by SAVIMEX using a procedure developed in collaboration with OCA using a microscope and a micro-sensor for the roughness, and a collimating lunette for the surface control (Fig. 6). The focal length $\mathrm{F}$ is related to the fiber numerical aperture NA and the beam diameter D. The best coupling efficiency was given by Zemax for $N A . F / D=0.46$, taking the telescope obstruction into account.

\subsubsection{OPD control}

This system was designed by OCA, and manufactured and tested at the Technical Division of the Institut National des Sciences de l'Univers (DT/INSU, Paris). The static OPD in $K$ is controlled with a $4 \mu \mathrm{m}$ accuracy within a few $\mathrm{mm}$ range. While the static OPD is adjusted for a given wavelength in each band, there exists an OPD drift between wavelengths. AMBER needs 
to correct this differential OPD in $J$ - and $K$-bands respective to the $H$-band (ESO fringe sensor unit functioning) during the observations. This chromatic OPD due to the atmospheric refraction is introduced during the telescope pointing. It is given by: $B \sin z\left(n\left(\lambda_{1}\right)-n\left(\lambda_{2}\right)\right)$, where $n(\lambda)$ is the refractive index of the atmosphere, $B$ is the baseline, and $z$ the zenithal angle. Considering the extreme case for which $B=200 \mathrm{~m}$ and $z=60^{\circ}$, the necessary OPD range is about $30 \mu \mathrm{m}$ in $K$ and $70 \mu \mathrm{m}$ in $J$. Such adjustments are achieved through drifts of the entire AMBER $K, H$, or $J$ spatial filter entrance parts. They are performed every few minutes.

Nevertheless, such translations cannot compensate for any chromatic OPD gap present inside each spectral band ( $\delta \lambda$ equal to $33 \mathrm{~nm}$ in $K, 32 \mathrm{~nm}$ in $H$, and $24 \mathrm{~nm}$ in $J$ ). This chromatic OPD gap is introduced when a difference in the glass thickness or in the fiber length between two interferometric arms exists. Limiting this relative thickness to $0.5 \mathrm{~mm}$, the contrast factor is thus ensured of being less than 0.99 . The relative fiber lengths in all the spatial filters were controlled by LAOG (Grenoble). It was shown that at the minimal resolution of AMBER, the contrast degradation factor due to differential dispersion is better than 0.99 inside each spectral band (Robbe-Dubois et al. 2003).

\subsubsection{Rapid OPD variations}

Instabilities of the VLTI beams can generate rapid achromatic and chromatic OPD changes. The first type of problem results in a deviation of the entire fringe pattern sideways. This is compensated by the VLTI itself. The chromatic changes degrade the interference pattern, curving the fringe shape at the timescale of these instabilities. It leads to the presence of a blurred pattern, especially at the sides of each spectral band (even if the OPD is well stabilized at the central wavelength of each spectral band). The main source of such instabilities comes from the differential positioning shifts of the VLTI beams, combined with the travel of light towards wedged dispersive glasses. Consequently, the requirement is to ensure that dynamic chromatic OPD rms values are lower than the uncertainty that comes from the fundamental noise levels. The goal is to reach the (mainly photon) noise corresponding to a 1-min measurement with a 5-mag star (observing with 2 UTs and the AO of the VLTI). In terms of chromatic OPD, between the central wavelength and the wavelength at the side of the considered bandwidth (most demanding case: $R=35$ ), this translates into OPD(noise) $=5.8 \mathrm{e}^{-11} \mathrm{~m}$ in $K$. Vannier et al. $(2002,2004)$ performed a complete study that led to the instrument requirement analysis concerning the wedge angle of the prismatic optics, and the surface quality and the relative thickness of the dispersive elements located prior to the fibers. This study included elements such as optical fibers, polarizers, and dichroics, but also those of the auxiliary modules described below in this paper, such as the Neutral Densities (NDN), the Matrix Calibration System (MCS), and the Atmospheric Dispersion Corrector (ADC).

\subsubsection{Dichroics}

The dichroics satisfy the photometric requirements: $\geq 0.95$ in reflection in the highest spectral band and $\geq 0.90$ in transmission in the lowest spectral band. An absorption is present at the end of the $H$-band (transmission from $86 \%$ at $1800 \mathrm{~nm}$ to $80 \%$ at $1850 \mathrm{~nm}$ ), but it does not affect the global throughput.

\subsubsection{Polarization control}

The following was done to minimize the polarizations' effects:

- Use the same number of reflections/transmissions between the 2 interferometer beams.

- Require identical coatings for the optics with the same functions between 2 beams: same substrate, same structure for the different layers, simultaneous manufacturings.

- Use polarization-maintaining fibers.

- Select one polarization direction at the fiber entrance.

- Control the incident angle on reflecting optics with an accuracy better than a fraction of a degree.

Prior to the spatial filters, the polarizers select one polarization direction to get rid of the cross-talk inside the fibers and of the phase difference between beams (variable differences during the telescope pointing). The selected direction is that which is not affected by the multiple reflections inside the instrument (perpendicular direction to the beam propagation plane).

Each polarizer is associated with one blade. The orientation of the two elements is mechanically controlled to ensure the direction of the light beam relative to the optical axis. The air blade located between the two prism constituents of each polarizer must be parallel for the optical system to respect the chromatic dynamic OPD specifications (see Sect. 3.1.4).

The relative polarization control also has an impact on the optical coating quality, in particular for the dichroics elements. All the optics with the same functions are simultaneously coated by the manufacturer, in particular the dichroics and the injection parabola located between the polarizers and the fiber entrance, to reach a flux difference of a few $\%$ after each reflection or transmission and a minimal phase difference generated by the different layer thicknesses.

At the spatial filter exits, an error on the dichroics layer thickness could generate a contrast degradation. The number of elements being small (1 reflection/arm in $K, 1$ reflection/arm and 1 transmission/arm in $H$, and 2 transmissions/arm in $J$ ), the overall effect is almost null. From Puech \& Gitton (2005): a typical $2 \%$ of error on the layer thickness generates less than $1 \%$ contrast loss in $K$. The neutral axes at the fiber entrance are controlled with a $\pm 3^{\circ}$ accuracy to compensate for the polarization direction rotations generated by the residual manufacturing differences between dichroics and by the incident angle differences. This ensures the maximum coherent energy inside the fibers. At the fiber exit, the differential polarization between beams before combining is controlled to within a few degrees.

\subsubsection{Acoustic perturbations}

Acoustic perturbations (due to step-by-step motors for instance) can modify the behavior of optical fibers that are sensitive to pressure variations. It can be shown that a typical talk produces a $60 \mathrm{~dB}$ acoustic intensity, which implies a phase instability of $10^{-7} \mathrm{rad}$, far below the specification (Perraut, internal report). To avoid disturbing other VLTI equipment in the interferometric laboratory, each instrument does not generate acoustic noise in excess of $40 \mathrm{~dB}$ at $2 \mathrm{~m}$ in all the directions.

\section{From the spatial filters to the detector}

The general parameters of the modules from the spatial filters to the detector are given: beam configuration, pupil diameter and separation, spectral resolution, and signal sampling. The modules are then described in details. 


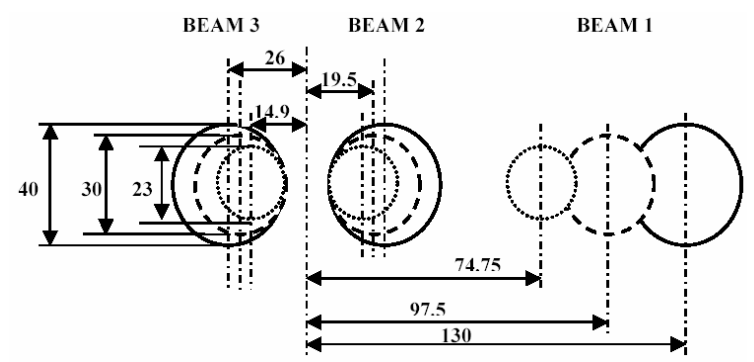

Fig. 7. AMBER pupil configuration in $J, H$, and $K$ with no anamorphosis.

\subsection{General parameters of the system modules}

Beam configuration: AMBER is a "dispersed fringes" instrument operating in the image plane. The three-pupil configuration (Fig. 7) is a non redundant line set-up. The maximal baseline is larger than the minimal one by a factor three. This pupil configuration produces three systems of fringes corresponding to the following baseline: $B_{\mathrm{m}}, 2 B_{\mathrm{m}}, B_{\mathrm{M}}=3 B_{\mathrm{m}}$ (the indices $\mathrm{m}$ and $\mathrm{M}$ mean minimum and maximum, respectively).

Pupil diameters and spectral resolution: the pupil size depends on the spectral resolution and on the number of grooves per $\mathrm{mm}$ of the grating. The central wavelength $\left(\lambda_{0}=2.2 \mu \mathrm{m}\right.$ in the $K$-band) involves a limitation of the number of lines per millimeter for the spectrograph grating (about 500 lines $/ \mathrm{mm}$ ). To optimize the recorded flux, the spectral channel is a bit undersampled $\left(\lambda_{0} / D\right.$ on one detector pixel). To obtain a spectral resolution of 10000 , the pupil diameter in the $K$-band is $D=40 \mathrm{~mm}$. The fringe sampling is the same for all the spectral bands. This implies smaller pupil diameters in the other spectral bands ( $30 \mathrm{~mm}$ for the $H$-band and $23 \mathrm{~mm}$ for the $J$-band). The instrument pupil is set by a cold stop inside the spectrograph. This pupil plane is combined with the neutral point of the cylindrical optics, roughly superimposed to the pupil masks located after the collimating parabola at the exit of the spatial filters. The precision of this conjugation has a negligible impact on the performance of this single mode instrument with a field of view limited to an Airy disk.

Pupil separation: in the optical transfer function (OTF), the fringes with the lowest frequency produce a coherent energy peak close to the incoherent energy peak (Fig. 8). To avoid the center of this fringe peak to be affected by the central single pupil peak, the minimal baseline $B_{\mathrm{m}}$ is: $B_{\mathrm{m}}>D\left(1+\lambda_{\mathrm{M}} / \lambda_{\mathrm{m}}\right)$. For a full band observation in the $K$-band $\left(\lambda_{\mathrm{m}}=2.0 \mu \mathrm{m}\right.$ and $\left.\lambda_{\mathrm{M}}=2.4 \mu \mathrm{m}\right), B_{\mathrm{m}}$ must be greater than $1.2 D$. For this reason, the distances between the three pupils are 1.3D, 2.6D, and 3.9D.

Signal sampling: anamorphosis factor and camera focal length: considering data reduction requirements, it is necessary to sample the fringes produced by the combination of the furthest beams by about four pixels on the detector. Each spectral element $\left(\lambda_{0} / D\right)$ is analyzed by one pixel. The magnification of the beams between the spatial direction and the spectral direction must be different. This anamorphosis factor $a$ is given by: $a=4 B_{\mathrm{M}} / D=15.6$. The size $p$ of the detector pixel, equal to $18.5 \mu \mathrm{m}$, is linked to the camera focal length $f_{\mathrm{c}}$ by the relation $4 p=a f_{\mathrm{c}}\left(\lambda_{0} / B_{\mathrm{M}}\right)$. The $f_{\mathrm{c}}$ parameter is then deduced from: $f_{\mathrm{c}}=p /\left(\lambda_{0} / D\right) \approx 350 \mathrm{~mm}$.

\subsection{Anamorphoser system (ANS)}

At the exit of the spatial filters, the beams enter the cylindrical optics anamorphoser (ANS) before entering the cold

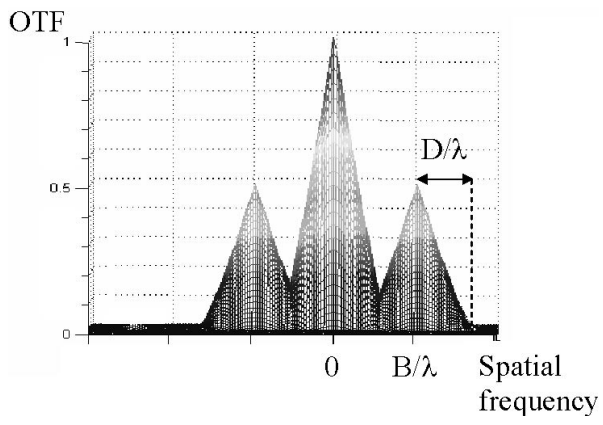

Fig. 8. Illustration: simulated optical transfer function (OTF) in which the coherent and incoherent energy peaks are not completely separated. The pupil separation is chosen such that the fringe peak center is not affected by the incoherent peak.

spectrograph (SPG) through a periscope and a focalizing optical component adjusting both axes and image positions at the interface between the warm optics and SPG. The anamorphoser is a Chretien hypergonar made of an afocal system of two cylindrical mirrors inserted in a parallel beam section. The anamorphosis factor, 15.6, is the ratio between the focal length of the two mirrors. The anamorphosis direction is perpendicular to the baseline. Such an afocal system contains a neutral point, located nearby after the focal plane of the smallest (2nd) cylindrical mirror. By putting the conjugate of the spectrograph cold stop at this neutral point, we avoid having the ANS introduce a difference between the longitudinal and transverse pupil positions, minimizing the aberrations and improving the cold stop baffling. The difficulty lay in the manufacturing of the 1st optics of the ANS shared by the 3 interferometric beams: a conic $220 \times 50 \mathrm{~mm}$ rectangle with a $2-m$ curvature radius in its length direction. The PTV optical quality of $633 \mathrm{~nm} / 5$ was tested on a specific optical bench at OCA.

\subsection{The spectrograph (SPG)}

The cold spectrograph SPG includes the following functions:

- Filtering of thermal radiation at the input image plane.

- Formation of a parallel beam.

- Accurate spatial filtering of the pupils.

- Separation of interferometric beams from photometric beams.

- Spectral analysis at three resolving power values.

- Formation of images on the detector plane. The list of the SPG is given in Table 2, with the product tree definitions and functions.

The optics and the optical bench are contained in a vacuum tight cryostat that allows the cooling of all functions to the working temperature of about $77 \mathrm{~K}$ by means of liquid nitrogen at atmospheric pressure. The spectrograph cryostat does not lodge the detector that stays in a second cryostat; the two cryostats are mechanically coupled, they share the same vacuum and work at the same temperature, but have two independent cooling systems. The coupling is achieved by a flexible bellow and an interface structure that allows for a certain degree of angular and linear adjustment to align the detector itself to the spectrograph optics. The optical design of SPG (Fig. 9) follows the general pattern of the grating spectrograph, with the necessary modifications dictated by the optical and mechanical accuracy requested by interferometry. A more detailed description of SPG is in Lisi (2003). 
Table 2. List of AMBER spectrograph modules with acronym definition and functions.

\begin{tabular}{ll}
\hline \hline Optical elements & Functions \\
\hline SPG-INW & Input slit. \\
Input Wheel & Image cold stop. \\
\hline SPG-CSY & Spectrograph optics cooling. \\
Cooling System & $\begin{array}{l}\text { Vacuum, temperature, and nitrogen } \\
\text { level control. }\end{array}$ \\
& Thermal flux reduction. \\
\hline SPG-ISD & Cold stop for the spatial \\
Imaging and Stopping & filter mode (thermal flux reduction). \\
Device & Technical operations (wide diaphragm). \\
& Calibration (dark). \\
& Beam collimation. \\
\hline SPG-PMW & Thermal flux reduction. \\
Pupils Masks Wheel & Beam size definition. \\
\hline SPG-IPS & Thermal flux reduction. \\
Interferometric Photometric & Beam splitting. \\
Splitter & Beams deviation. \\
& Photometric images deflection on DET. \\
\hline SPG-DIU & Spectral dispersion. \\
Dispersion Unit & Spectral wavelength selection. \\
& Spectral resolution selection. \\
& Spectral modulation. \\
\hline SPG-CHA & Beam combination. \\
Camera & Beam focalization. \\
& Sampling. \\
\hline
\end{tabular}

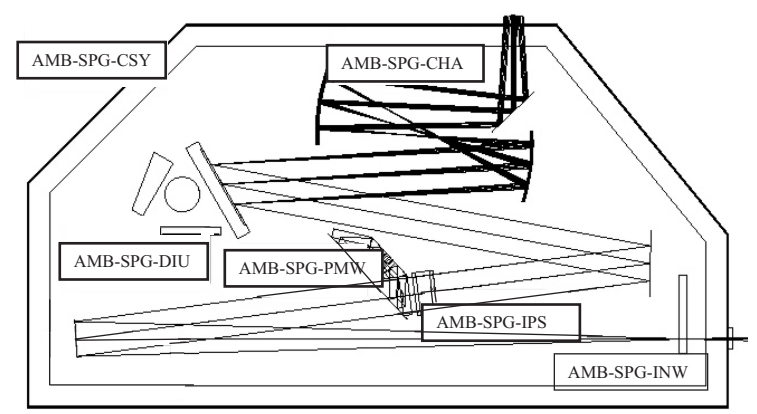

Fig. 9. Optical design of the SPG. For the acronym definition see Table 2.

\subsubsection{SPG optical configuration}

The OPM-OSI module (Fig. 2), located at the side of the SPG cryostat, injects the beam into the spectrograph through a $\mathrm{CaF}_{2}$ window. The beam is spatially filtered by the slit, mounted on a wheel that also carries several components useful to the calibration and alignment operations.

A collimating mirror produces the parallel beam. The collimating mirror is a symmetric paraboloidal mirror (with respect to the focal point) of the focalizing mirror; this configuration has the purpose of minimizing the optical aberrations. It has a focal length of $700 \mathrm{~mm}$ and an aperture of $\mathrm{F} / 3$.

The wheel SPG-PMW carries the pupil masks, located on the plane of the pupil image, which help to minimize the stray thermal background. The optical component SPG-IPS is a beam splitter that steers part of the flux coming from each telescope from the parallel beam for photometric calibration, leaving the largest fraction of the flux for the interferences. The beams that carry the photometric information run along the same optical path as the interferometric beams, but they are suitably directed to form separate images on the detector.
For the purpose of spectral analysis, a rotating device (SPG-DIU) allows for the choice among three dispersing components: two gratings $(497 \mathrm{~g} / \mathrm{mm}$ and $75 \mathrm{~g} / \mathrm{mm}$ for respective resolutions of 10000 and 1500), and a prism. The support of the gratings and the prism is motorized to allow us to select the spectral range and resolution. The angular accuracy of this support is about 3 pixels of the detector $\left(\approx 30^{\prime \prime}\right)$, which implies a spectral calibration procedure.

The final optical function before the detector is the camera SPG-CHA, designed around three mirrors. It is composed of two aspherical mirrors with a total focal length of $350 \mathrm{~mm}$ (see Sect. 4.1.4) and an aperture of about F/2. A plane mirror steers the beams coming from the camera unit to send it to the DET cryostat. A spectral filter is inserted in the $J$ pupil mask to eliminate the background coming from the $K$-band, while observing with the second spectral order of $J$.

\subsubsection{Opto-mechanics and performance}

The requirements for the optical design reflect the need to mount all the optics inside a cryostat, where the accessibility for alignment is reduced and the displacements of components after the cooling are very large. The tolerance analysis shows a fringe contrast degradation factor of about $95 \%$ under the following constraints: a total positioning error of the optical elements of $0.1 \mathrm{~mm}$, an angular positioning error (tilt) of $2.3 \mathrm{mrad}$, and a quality of optical surfaces better than 5 fringes in focusing and 1 fringe in irregularity. This performance depends only on the use of the detector position along the optical axis as a compensator, with a total displacement of less than $2.3 \mathrm{~mm}$ with respect to the nominal position. The surface quality (microroughness) of the metallic mirrors has an impact on the efficiency. The machining of the aspherical and plane mirrors allowed us to produce the respective roughnesses of less than $10 \mathrm{~nm}$ and $5 \mathrm{~nm} \mathrm{rms}$, to ensure that the loss of light be less than $1.5 \%$ on each mirror. One feature of the opto-mechanical design is keeping the optics aligned at room temperature and at liquid nitrogen temperature without adjustments. Tests of the SPG optics either at room or at operative temperature showed no significant differences of performance between the two sets of measurements, confirming the design concept.

\subsubsection{Vacuum and cryogenic system}

The whole system is lodged inside a vacuum-tight case made out of welded steel plates with suitable reinforcing ribs. The liquid nitrogen vessel is a box-like structure (worked out of a single aluminum block completed by a welded cover), whose bottom plate is the cold optical bench. The external case supports the cold bench by means of an hexapod (composed of six fine steel beams), dimensioned to allow the SPG system to be placed on a side without undergoing permanent deformation. All the optics are enclosed in the radiation shield, in tight thermal contact with the cold bench. The model of the thermal behavior shows that all its points are at most two degrees over the cold bench temperature; this is confirmed by measurements. The SPG optical system includes a total of three moving wheels, the aperture wheel, the pupil mask wheel, and the grating wheel. To simplify the engineering, these three functions are implemented by cryogenic motors (Berger Lahr 5-phase), modified according to the ESO experience. Positioning of the associated wheels is performed by a worm-wheel gear that is substantially irreversible and acts as a stop to the force exerted by the spiral spring used to overcome 


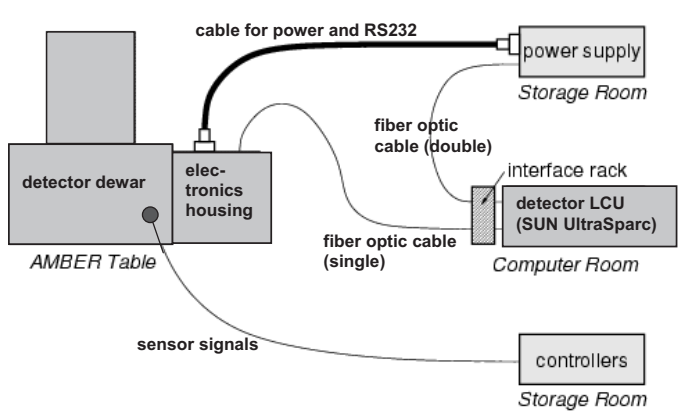

Fig. 10. Physical overview of the AMBER detector hardware.

the backlash. The temperature of the three motors is constantly monitored by the control system using local Pt100 sensors. The nitrogen vessel can store up to $19 \mathrm{dm}^{3}$ of liquid $\mathrm{N}_{2}$, while the mass of aluminum to be cooled is about $24 \mathrm{~kg}$. The design total thermal load is of the order of $25 \mathrm{~W}$. A turbo-molecular pump establishes the operative vacuum and in normal operation, a small quantity of active charcoal keeps the internal pressure at the level of $2 \times 10^{-5} \mathrm{mbar}$ for several months. The liquid nitrogen supply lasts for about $30 \mathrm{~h}$.

\subsection{The detector (DET)}

\subsubsection{Hardware overview}

The detector is located in a dewar that is cooled down to $77 \mathrm{~K}$ with liquid nitrogen. The detector electronics housing is directly attached to the dewar to avoid electronic interference resulting from long signal paths (Fig. 10). It is connected to the sensor by two short cables. In addition to this, it complies with challenging constraints concerning heat dissipation, interference, and electromagnetic compatibility, for example. The power supply is not installed close to the detector electronics housing. A distance of a maximum of $15 \mathrm{~m}$ is allowed between the electronics housing and the power supply. The connection is made by a single power cable. This cable also contains the galvanically isolated RS232 serial line for controlling the electronics. The power supply rack is installed in the instrument control cabinet in the storage room. The digital image data is transmitted to the detector LCU via a fiber optics cable.

\subsubsection{Functional overview}

The detector electronics consists of the following modules:

1. An infrared detector (HAWAII-1 focal plane array from Rockwell).

2. A sequencer generates clock patterns necessary for reading and sampling the sensor. It can be configured by data sent through the galvanically isolated serial line (RS232). It also generates a header containing information about image format, readout mode, etc.

3. A clock driver boosts the digital signals from the sequencer.

4. A video amplifier supplies all necessary bias voltages and prepares the analog signal from the IR sensor for sampling.

5. An analog to digital converter ADC samples the analog signal. On the ADC board there is also digital logic for averaging several samples (subpixel sampling). A fiber optical transmitter on the same board feeds the image data into a fiber optics cable connected to the detector LCU.

6. A power supply.

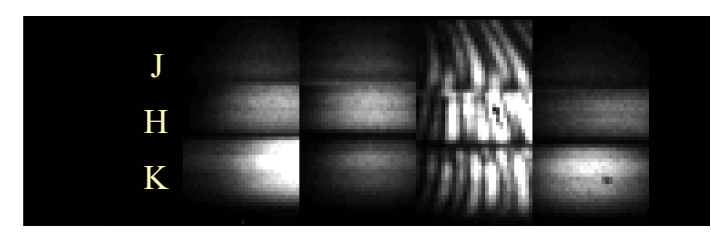

$$
\text { P1 P2 In P3 }
$$

Fig. 11. Image with the CAU light: dark current, photometric beams $\left(\mathrm{P}^{*}\right)$, and interferometric beam (In).

\subsubsection{Detector characteristics}

Detector type: Rockwell HAWAII-1 FPA (focal plane array, one quadrant in use)

Detector size: $512 \times 512$ pixels

Pixel size: $18.5 \mu \mathrm{m} \times 18.5 \mu \mathrm{m}$

Operating temperature: $77 \mathrm{~K}$

Quantum efficiency: $>50 \%$ for $1-2.4 \mu \mathrm{m}$ wavelength

Other properties of the detector chip were measured and are listed below:

- detector number: \#159;

- full well capacity: 63670 e-;

- $1 \%$ nonlinearity: $26289 \mathrm{e}-$;

- conversion factor: $4.70 \mu \mathrm{V} / \mathrm{e}$-;

- readout noise (CDS @ 500 kHz): $11.6 \mathrm{e}-$;

- number of bad pixels: 1489 ;

- clusters of bad pixels $(\geq 4): \approx 10$.

\subsubsection{Image on the detector}

Figure 11 shows one image recorded on the detector with the CAU light. From left to right are visualized the dark current, two photometric beams, the interferometric beam, and the 3rd photometric beam. The flux in the $J$-band was not optimized.

\section{Auxiliary modules}

\subsection{Remote artificial sources (RAS) and calibration and alignment unit (CAU)}

Artificial sources are provided in the module RAS for alignments in the visible, flux and OPD control, contrast, and spectral calibrations. These sources are: one laser diode and one halogen lamp allowing alignments and calibration of the matrix of the "pixel to visibility" linear relation (P2VM). The halogen source feeds two different single-mode fibers, one dedicated to the $K$-band, the other to the $J$ - and $H$-bands, to transport the light up to the CAU, which can simulate the VLTI in the integration and test phase (Fig. 12). The use of the same fiber in $J$ and $H$ is a compromise solution allowing us to save space and money without losing too much (a few tens of a \%) injected light in both bands. The exits of the two fibers coming from the RAS provide almost point-like sources in $J, H$, and $K$.

The retained optical configuration of the CAU chosen on the basis of generating an achromatic path length uses a wavefront division configuration. The spectral beams exiting the RAS fibers are collimated and recombined in a global wavefront. The latter is magnified and divided in three parts via a set of plane mirrors to be injected inside AMBER via a movable $45^{\circ}$ mirror. The equivalent $K$-magnitude of the CAU was estimated to be -1.4 for the medium beam of AMBER and 0 for the extreme beams (different because of the Gaussian distribution of the global wavefront). The instrumental contrast generated with 


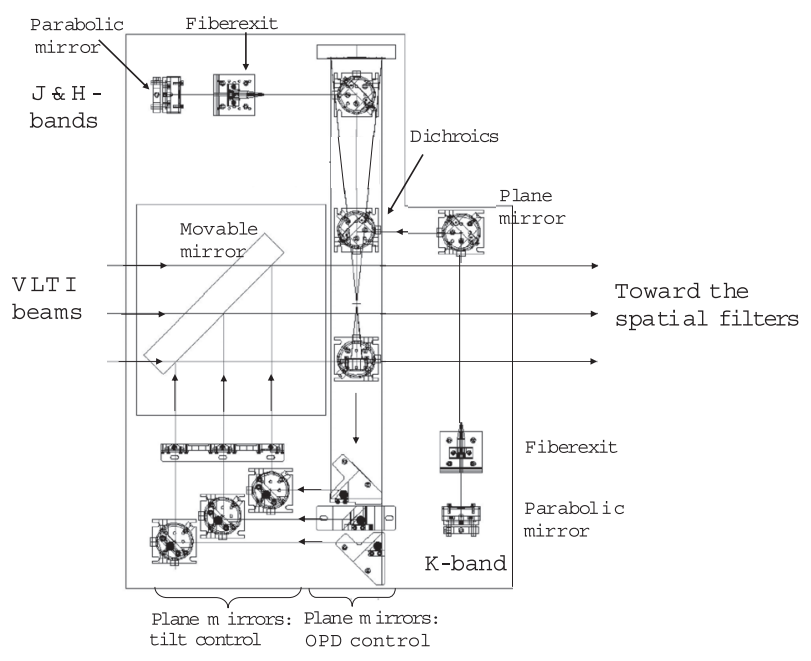

Fig. 12. Calibration and Alignment Unit (CAU).

the CAU light is not $100 \%$. Given that the cores of the fibers have a finite dimension, the CAU does not provide perfect unresolved sources. Nevertheless, this contrast (from 0.75 to 0.87 depending on the spectral resolution and baseline) is taken into account in the P2VM procedure and does not affect the instrumental contrast of AMBER illuminated by the VLTI.

The CAU light is also used to perform a calibration of the medium spectral resolution. A blade can be inserted at the spectrograph entrance generating Perot-Fabry-like interference fringes with a $30 \%$ contrast and a periodicity of about $0.05 \mu \mathrm{m}$. In low spectral resolution, we use the spectra as they are defined by the three $J, H$, and $K$ dichroics transmission curves and calibrated with spectroscopic reference stars and lamps. We successively observed the spectrum of each individual spatial filter by closing the shutters of the two others, which yields a subpixel calibration with an accuracy of about $0.01 \mu \mathrm{m}$, enough for this low resolution mode. The highest spectral calibration mode can only be calibrated using spectroscopic calibrators and/or telluric lines like any other high resolution infrared spectrograph.

\subsection{Calibration system}

To calibrate the P2VM it is necessary to introduce a controlled phase delay (between $60^{\circ}$ and $120^{\circ}$ ) between the interferometric arms. In the present state of the instrument, the piezoelectrics used for the chromatic OPD control are accurate enough (a few nanometers) to perform this phase delay. Nevertheless, a requirement of $10^{-4} \mathrm{rad}$ on the repeatability of the phase value was initially defined to reach some differential interferometric goals. To achieve this performance, a specific set-up (MCS) was designed and could be used in a near future if necessary. It consists of couples of slighty inclined glass blades placed in each beam path, the second one having a tilt opposite to the first one (leading to a V glass shape in each beam path). This system allows for very good tolerance on the exact absolute thickness of the blades, as their thickness variations will be compensated for through a slight inclination control during the optics mounting. This type of configuration enables a repeatable phase delay even if the system undergoes some inclination during its positioning.

\subsection{Atmospheric dispersion corrector (ADC)}

The role of the ADC is to correct the differential transversal dispersion of the atmosphere in $J$ and $H$. The specific constraints

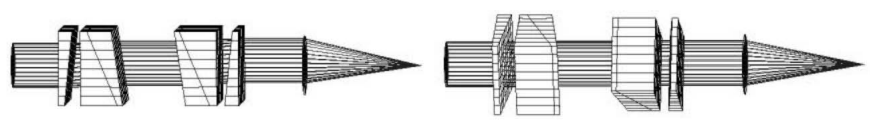

Fig. 13. Relative axial rotation of one ADC system relative to the other one at $z=0^{\circ}$ and at $z=60^{\circ}$.

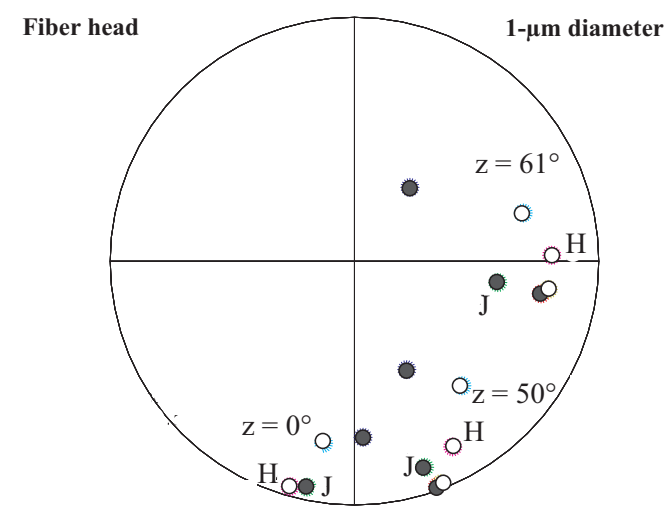

Fig. 14. Combined optical and positioning errors of the ADC. The spots represent the impacts of the beam for 3 wavelengths inside each band, $J$ (black spots) and $H$ (white spots), for 3 values of the zenithal angle $z$, and taking into account the following errors: prism angles manufacturing, error on the prism gluing, sensitivity of the axial rotation of the two glued prisms respective to the first one, tip/tilt of this rotation axis, tip/tilt of the 3-prisms assembly, sensitivity of the $360^{\circ}$-rotation of the two ADC prism system, and inclination induced during this rotation. The worse expected coupling degradation factor is 0.85 in $J$ and $H$.

of AMBER require a different conception than the Risley prism generally used. The original system of AMBER is composed of two sets of 3 prisms rotating with respect to each other (Fig. 13) and inserted in each beam prior to the $J$ and $H$ spatial filters. Each system is composed of a first prismatic blade in BK7 and of a doublet of blades in SF14 and F2 glued together. During an observation with the UTs of objects located from $0^{\circ}$ up to $60^{\circ}$ from zenith, the correction of the transversal dispersion is performed by an axial rotation of the two systems. During this rotation, the maximum image shift due to combined optical manufacturing and positioning errors is less than $1 \mu \mathrm{m}$ on the entrance fiber heads so as not to affect the coupling efficiency by more than $15 \%$ in $J$ and $H$ (Fig. 14). This coupling loss is taken into account in the throughput budget.

\subsubsection{Bypass (BYP)}

A specific system (BYP) with a commutable mirror was designed to bypass the spatial filters so that preliminary mechanical adjustments and optical alignments in the visible could be performed with technical tools (CCD, lunette) and injected towards the final detector (DET) via the spectrograph. It is also used to ensure that the CAU fibers for the $J H$-band and the $K$-band are superimposed. It appears that the BYP can be used on sky for acquisition of complex sources or technical controls during VLTI troubleshootings. The sampling is 23 mas/sky with UTs and the non-vignetted field about 1 as.

\subsubsection{Neutral densities (NDN)}

Two sets of NDN can be inserted in the VLTI beams to avoid the detector saturation. One set is chosen as a function of the target brightness. Two flux attenuations are possible: 10 and $10^{2}$. 


\section{Optical study and present performance}

The performance of AMBER is given by the signal-to-noise ratio $(S N R)$ of the visibility derived from AMBER interferograms. Malbet et al. (2003) showed that the instrument contrast $V_{\text {inst }}$ must be $80 \%(90 \%)$ and the optical throughput $t_{\mathrm{A}} C_{\text {eff }}$ larger than $2 \%(5 \%)$ to reach the magnitude specification (goal) defined for a fringe detection at $S N R=5$. The parameter $t_{\mathrm{A}}$ includes both optical transmission and the fiber coupling degradation factor due to misalignments. $C_{\text {eff }}$ is the fiber coupling efficiency taken to be about $81 \%$, considering the telescope obstruction. The other considered parameters in the $S N R$ calculation are:

- $E_{0}$ : flux of a zero-magnitude star at the considered wavelength;

- $m$ : expected magnitude of the observed object;

- $S$ : telescope surface area (considering a telescope diameter of $8 \mathrm{~m}$ for the UTs and $1.8 \mathrm{~m}$ for the ATs);

- $t_{V}$ : VLTI optical throughput (20\% in $J, 26 \%$ in $H$, and $30 \%$ in $K$ );

- $S R$ : Strehl Ratio. The $S R$ in $K$ for an on-axis reference source is equal to $50 \%$ (when the science and reference sources are 1 arcmin away, the Strehl in $K$ is divided by two);

- $\Delta \lambda$ : spectral bandwidth;

- $\tau$ : elementary exposure time $(\tau=10 \mathrm{~ms}$ for the high accuracy mode, $50 \mathrm{~ms}$ for the high sensitivity mode, and up to $100 \mathrm{~s}$ for the long exposure mode);

- $\eta$ : detector quantum efficiency (0.6);

- $n$ : number of pixels for one visibility measurement (about 16 for 3 telescopes).

The computation of the magnitude assumes the use of a single polarization. The integration time $\tau$ depends on AMBER observing modes (Malbet et al. 2003).

In this section, we present the allocations for the contrast and throughout of AMBER with the associated errors budgets. The results of the optical study and the expected instrumental stability are compared with measurements in laboratory. Then the results of the two commissionings at VLTI are discussed.

\subsection{Allocations for the contrast and throughput of AMBER}

The diagram of Fig. 15 summarizes all the identified contributions to the throughput and instrumental contrast ( $B \sin z=51.2 \mathrm{~m}$ ). More details on the allocations are given in Table 3 . The throughput results are based on conservative values from manufacturer information on coatings. The next sections give the error budgets on the fiber coupling and the contrast.

\subsubsection{Error budget on the fiber coupling}

This paragraph gives a list of the errors that can contribute to the degradation of the fiber coupling $C_{\text {eff }}$. To derive this error budget, analytical simulations were performed (Escarrat 2000). These results were confirmed with the ray tracing tool Zemax in which the optical injection of light inside a fiber can be estimated (Wagner \& Tomlinson 2004).

Table 4 shows the error budget on the fiber coupling in terms of fiber axis inclination respective to the incident optical axis (tilt), lateral shift of the fiber head, and defocus compared with the localization of the injection optical focal point. Coupling efficiencies without misalignments were also estimated after modification of the ratio $F / D$, where $F$ is the injection optical focal length and $D$ the pupil diameter. This ratio depends on the

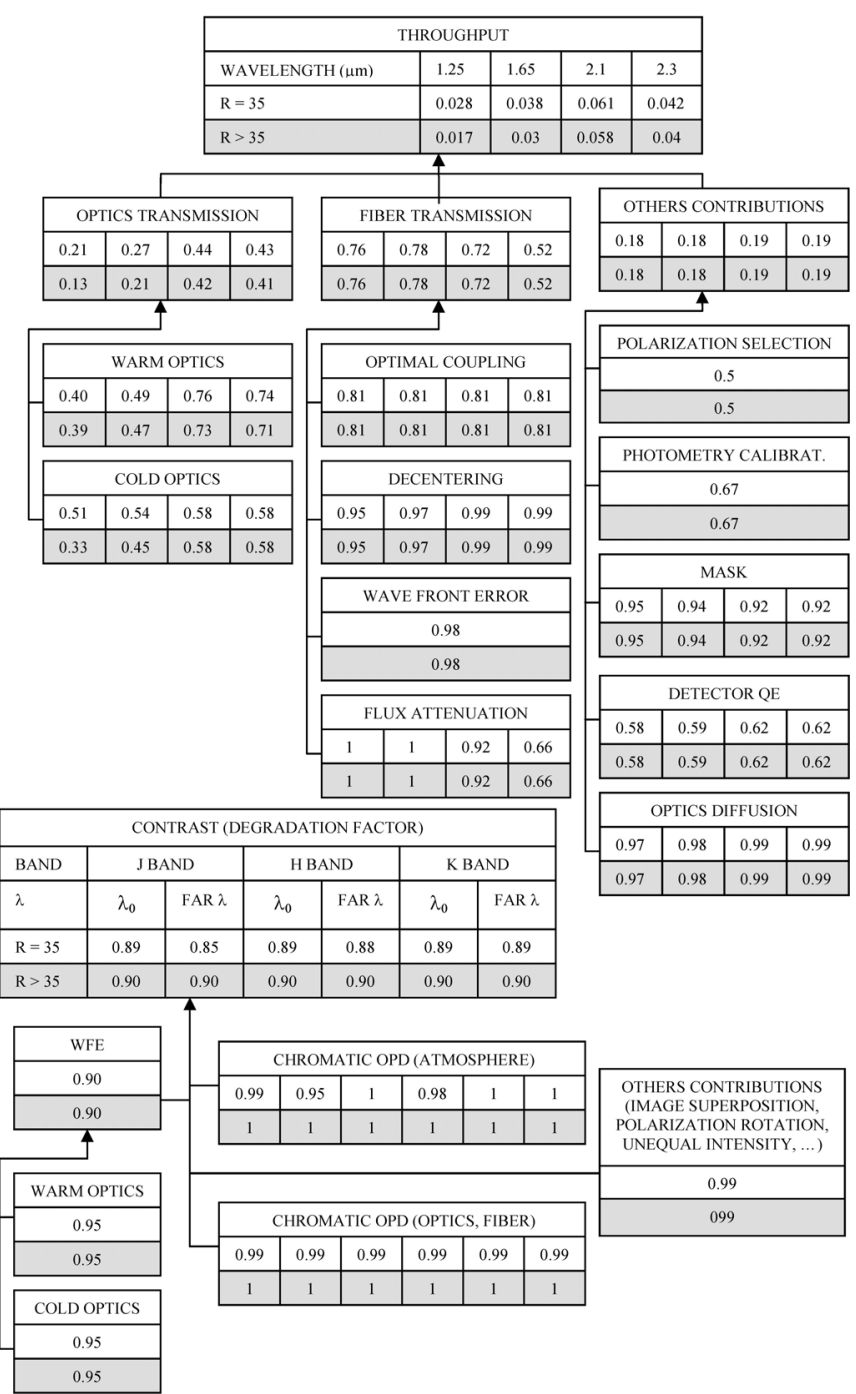

Fig. 15. Contributions to the throughput and instrumental contrast $(B \sin z=51.2 \mathrm{~m})$.

numerical aperture. The error budget on fiber coupling requirements was then compared to VLTI performance to validate the hypotheses. It was the input for the definition of the degrees of freedom necessary on each optical element located at the entrance of the fibers and to achieve their detailed tolerance analysis.

\subsubsection{Contrast error budget}

Results on contrast-error budget in the $K$-band (priority of AMBER) with a 35 spectral resolution (most demanding case) are shown in Table 5. This error budget concerns differential errors between interferometric beams. A degradation factor $\rho$ is such that the measured instrumental contrast $V_{\text {inst }}$ is equal to the ideal contrast value $V_{i}$ times this factor $\left(V_{\text {inst }}=\rho V_{i}\right)$. It was assumed in a first approximation that the errors were independent. Some of the requirements concerning static errors were analytically derived. Others requiring dynamical analysis needed some simulation. The latter considered a Gaussian wavefront going through a simple 2-telescope interferometer using the AMBER spectral bandwidths and resolutions. Four pixels were considered to sample the fringes. The considered pupil dimensions after anamorphosis in the horizontal direction were $40 \times 2.6 \mathrm{~mm}$ 
Table 3. Expected performance of the AMBER design based on the optical study described in this paper.

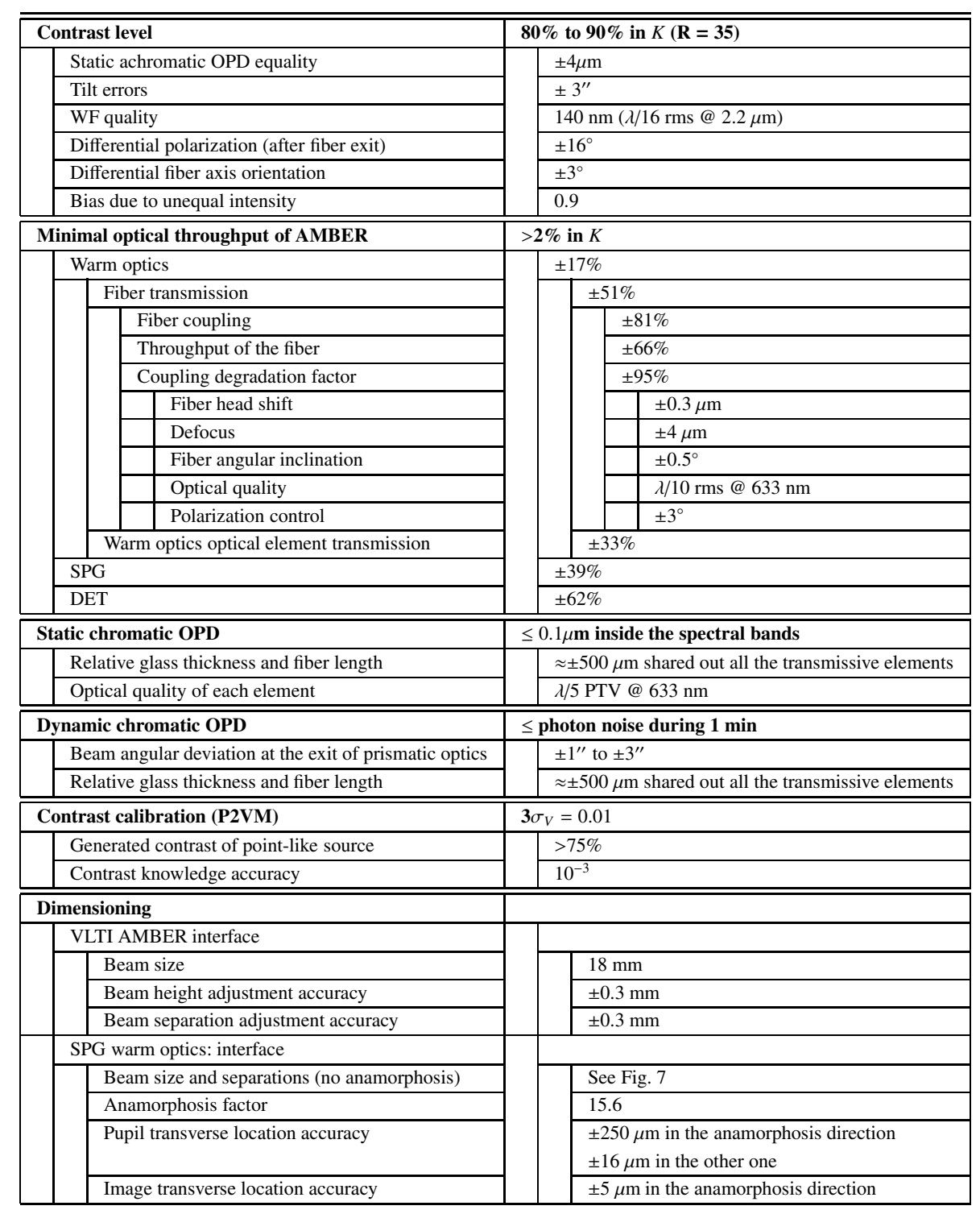

Table 4. Error budget on the coupling efficiency. $F=$ focal length of the injection optics, $D=$ pupil diameter.

\begin{tabular}{ccccc}
\hline \hline & $\begin{array}{c}\text { Deviation } \\
\text { from optimum }\end{array}$ & $1.10 \mu \mathrm{m}$ & $1.65 \mu \mathrm{m}$ & $2.20 \mu \mathrm{m}$ \\
\hline Tilt & $0.5^{\circ}$ & $>0.99$ & $>0.99$ & $>0.99$ \\
Fiber head shift & $0.3 \mu \mathrm{m}$ & $>0.98$ & $>0.99$ & $>0.99$ \\
Defocus & $4 \mu \mathrm{m}$ & $>0.99$ & $>0.99$ & $>0.99$ \\
$F / D$ & 0.93 & $>0.99$ & $>0.99$ & $>0.99$ \\
\hline Total & & $>0.95$ & $>0.96$ & $>0.96$ \\
\hline
\end{tabular}

in $K, 30 \times 1.9 \mathrm{~mm}$ in $H$, and $20 \times 1.5 \mathrm{~mm}$ in $J$. The simulation estimated the normalized optical transfer function (OTF), which consists of three peaks from which the fringe contrast can be evaluated: two high frequency peaks containing the coherent energy, and one low frequency peak containing the incoherent energy (Fig. 8). The fringe contrast was then defined as the ratio of the coherent to the incoherent energies. More details on the error definition are given in the Appendix. The results listed in Table 5 were used to define the degrees of freedom necessary
Table 5. Contrast degradation factors and allocations derived in the $K$-band with a 35 spectral resolution. $l_{\mathrm{c}}$ is the coherence length and Airy the Airy disk size.

\begin{tabular}{|c|c|c|c|}
\hline Allocations & $\rho$ & $\begin{array}{c}\text { Contrast } \\
\text { noise }\end{array}$ & $\begin{array}{l}\text { Requirement } \\
\text { rms }\end{array}$ \\
\hline OPD equality & 0.995 & & $l_{\mathrm{c}} / 19(4 \mu \mathrm{m})$ \\
\hline OPD vibration (rms) & & $10^{-3}$ & $\lambda / 140(16 \mathrm{~nm})$ \\
\hline Image overlapping & 0.99 & & Airy/10 (3") \\
\hline Overlapping jitters & & $10^{-3}$ & Airy/12 (3") \\
\hline Wavefront quality & 0.95 & & $\lambda / 16(140 \mathrm{~nm})$ \\
\hline Defocus & 0.99 & & $\approx 10 \mathrm{~mm}$ \\
\hline Phase delay & 0.99 & & $17^{\circ}$ \\
\hline $\begin{array}{l}\text { Differential rotation of } \\
\text { polarization frames }\end{array}$ & 0.99 & & $16^{\circ}$ \\
\hline Unequal intensity & 0.999 & & $I_{1} / I_{2} \approx 0.9$ \\
\hline Total & $\approx 0.89$ & $\approx 10^{-3}$ & \\
\hline
\end{tabular}

on each optical element located after the exit of the fibers and to achieve their detailed tolerance analysis. 


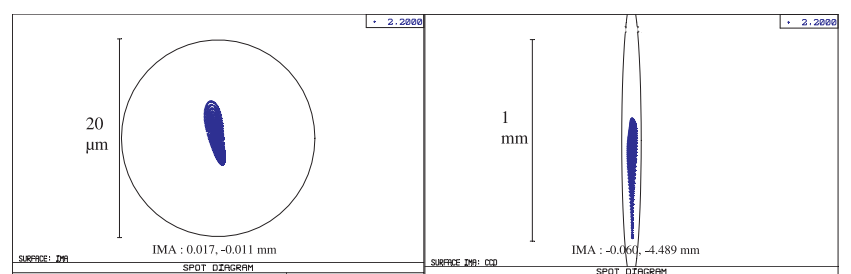

Fig. 16. Left: spot diagram at the SFK fiber entrance using the light from the VLTI. The coupling loss ratio is about $2.6 \%$. Right: spot diagram at the spectrograph entrance in the $K$-band. The associated Strehl ratio is 0.92 . This optical quality leads to a differential WF rms error of $\lambda / 15.5 @ 2.2 \mu \mathrm{m}$, which corresponds to a 0.95 instrumental contrast degradation factor.

\subsection{Optical study}

The optical configuration performance was assessed by the study of spot diagrams at the fiber entrance and by the estimation of the optical quality at the entrance of the spectrograph (Fig. 16). These respectively reflect the expected maximum fiber coupling efficiency (Table 4) and the expected maximum contrast (Table 5). The ray tracing optical tool Zemax was used to test the tolerance of all elements and surfaces. It is able to estimate a fiber coupling efficiency and to perform the tolerance analysis of the elements located before the fiber entrance such that the coupling stays very near a maximum value previously defined. Zemax is not able to estimate a fringe contrast for a configuration such as that of AMBER, but provides the wavefront (WF) optical quality taken into account in the contrast budget.

\subsubsection{Estimation of the optical throughput from the optical study}

Concerning the AMBER warm optics: the expected coupling efficiency degradation factor estimated from the tolerance analysis is $96 \%$ in $K$, including the VLTI WF errors. This, in addition to the throughput measurements provided by the optics manufacturers and to the coupling efficiency, leads to a global optical throughput $t_{\mathrm{A}} C_{\text {eff }}$ of $8 \%$ to $12 \%$, depending on the wavelength (VLTI included). Including the spectrograph and the detector efficiency in the estimations lead to a global throughput of $2 \%$ to $4 \%$ meeting the specifications.

\subsubsection{Estimation of the contrast from the optical study}

At the entrance of the spectrograph, the expected optical quality of one beam is expressed in terms of differential WF rms error: $\lambda / 15.5 @ 2.2 \mu \mathrm{m}$, which corresponds to a contrast degradation factor of 0.95 in $K$. This represents the instrumental factor to be applied to the fringe visibility, due to the WF quality of the AMBER warm optics located at the exit of the fibers. The contrast budget of the warm optics taking into account additional parameters such as achromatic OPD errors, differential chromatic OPD, global tilt errors, defocus, differential direction of polarization, and unequal intensity, is about 0.90 in $K$.

The wavefront aberration of the optical system from the input slit to the detector plane has a measured PTV value of 2.8 fringes @ 633 nm and a rms of about 200 nm (on the largest pupil area the system exploits). This value is well inside the error budget for SPG. The contribution to the fringe contrast degradation factor of AMBER coming from SPG is estimated to be greater than $98 \%$. Figure 17 shows the residual wavefront aberration at liquid nitrogen temperature as measured with
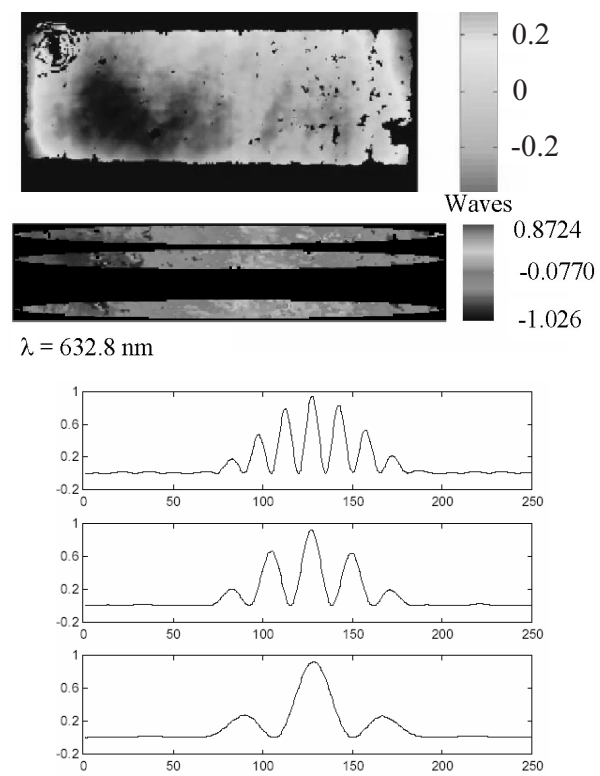

Fig. 17. Above: residual WF aberration at liquid nitrogen temperature as measured with external optical elements $(45 \times 16 \mathrm{~mm}$ surface). Center: three pupil masks superimposed to the measured wavefront. Below: simulated PSF at $2200 \mathrm{~nm}$ for the combinations of two apertures at different separation.

external optical elements, independently of the warm optics. The surface size is $45 \times 16 \mathrm{~mm}$. The pupil mask was then superimposed to the measured WF to simulate the final configuration and to estimate the associated PSF and fringe contrast. The evaluation of the AMBER instrumental contrast of $88 \%$ from the optical study is higher than the specifications.

\subsection{Stability}

To get very accurate phase and contrast measurements, the interferometer has to be as stable as possible. To observe fine structures in the disks and jets around a sufficient number of young stellar objects, it is necessary to obtain a requirement of 0.01 (Malbet et al. 2003) on the instrument contrast stability during $5 \mathrm{~min}$, the initial estimation of the calibration cycle. There are several sources of instabilities that need to be taken into account including: temperature gradients, vibrations, and microturbulence.

\subsubsection{Temperature gradients}

Temperature gradients can occur when the temperature is not correctly monitored or when heating (active motors, people, sources on, ...) or cooling (cooled dewar) sources are present close to the instrument. The global dissipation in the laboratory was measured (Puech \& Gitton 2005): $+50 \mathrm{~W}$ for motors and electronics and $-20 \mathrm{~W}$ for the cryostat. These internal fluxes are not a problem so far, but the effects on the highest goals still have to be analyzed.

The measured temperature gradient through the AMBER table, from the CAU to the spatial filter $J$, is currently $0.03 \mathrm{~K}$. It is stable over one night if there are no interventions in or near AMBER. The fastest fluctuations are lower than the current $0.01 \mathrm{~K}$ monitoring accuracy. This corresponds to predicted fluctuations of the differential visibility and phase smaller than $0.001 \mathrm{rad}$ (Vannier 2003). This is confirmed by our current 
measurements, which are currently limited to a few $0.001 \mathrm{rad}$ accuracy by the atmosphere. If the external conditions allow us to achieve the highest accuracy of $10^{-4}$ rad necessary for exoplanets spectroscopy (while using the ESO fringe sensor FINITO for example), the current thermal stability of AMBER of $10^{-3} \mathrm{rad}$ will prove to be insufficient. Vannier (2003) showed that a possible solution to reach such a goal would be to thermally insulate the AMBER fibers.

\subsubsection{Vibrations}

Vibrations, inducing OPD fluctuations, can occur inside the instrument when internal sources of vibration exist (motors, ventilation, nitrogen bubbling, water cooling devices, ...) and/or when external vibrations are transmitted to the instrument via the floor.

Optical tables can also act as cavity resonators according to their damping capabilities.

To address this, specific considerations (pneumatic feet, water chiller, isolation of water cooling pipes, use of screw-type pumps, placing all vibration generating equipment on soft pads) and an estimation of eigen frequencies were performed.

\subsection{Measurements in laboratory}

The performance of AMBER measured in laboratory (Rousselet-Perraut et al. 2004) for the Preliminary Acceptance in Europe (PAE) showed that the instrument reaches the requirement and approaches the goals. Although absolute photometric measurements could not be performed in laboratory as no dedicated bench was available, relative measurements gave a throughput of $5 \%$ in $K, 13 \%$ in $H$, and $10 \%$ in $J$. As regards to these values, limiting magnitudes of 11 could be reached. The instrumental contrasts measured in $K$ with the CAU were: 0.78 at Low Resolution (LR), 0.87 at Medium Resolution (MR), and 0.83 at High Resolution (HR). Let us not forget that the CAU sources are slightly resolved by AMBER. These values were taken in the presence of the polarizers. Removing them has the consequence to reduce the contrasts by up to few tens of $\%$. An accuracy of $1 \%$ on the CAU contrast at $2.2 \mu \mathrm{m}$ was measured, meeting the specification. The specifications of $10^{-2}$ over 5 min on contrast stability and of a few $10^{-3}$ rad over 1 min on differential phase stability are met. Additional measurements were performed with the CAU during the alignment integration and verification (AIV) phase at Paranal (Robbe-Dubois et al. 2004). The instrumental contrasts in $H$ and $J$ were very similar to those in $K$.

\subsection{Results of commissionings}

This paragraph summarizes the situation of AMBER after its integration (February-March 2004) and its first two commissioning runs (May and October 2004). More details are given in (Petrov et al. 2007). The 3rd commissioning took place in February 2006, its analysis is in progress and will be reported in http: //www-laog.obs .ujf-grenoble. fr/amber/.

AMBER is fully operational in 2 telescopes and 3 telescopes modes. It has been commissioned in $\mathrm{MR}$ in the $K$-band and in LR in the $K$ - and $H$-bands. For AMBER alone, the contrast transfer function is in the $65 \%-85 \%$ range depending on modes. The contrast, differential phase, and phase closure stability are of a few $10^{-3}$.

The flux collecting efficiency of the AMBER/VLTI system was higher than expected, in spite of the absence of the VLTI image sensor in the focal laboratory. This corresponds to a limiting magnitude higher than 11 in LR and higher than 7 in MR (without fringe tracker). So far we have been able to acquire objects and to detect fringes up to $K=9$ in LR and $K=7$ in MR. In MR, this is very close to the theoretical limit. In LR, the object acquisition was inefficient due to the absence of the ESO infrared image sensor in the focal laboratory at the time of the first commissioning runs. The implementation of the image sensor has dramatically improved the acquisition speed and the longer term stability of the injection, but has not radically changed the fiber injection efficiency in the minutes following the source acquisition.

The data processing of AMBER is operational. The delivered routines can be used as black boxes for applications in the $1 \%$ accuracy range, given that the appropriate detector calibration procedure has been used. In MR, there is no operational difference observing with 2 or 3 telescopes. In LR, with the appropriate VLTI OPD model (controlling the delay line stroke in each interferometric arm) and with a fringe search simultaneous for all baselines, the operation with $3 \mathrm{~T}$ is exactly as easy as the operation with $2 \mathrm{~T}$.

Almost all the data recorded with AMBER on the UTs is affected by a piston vibration problem coming mainly from the UT coude train and generated by several external sources such as the fans of the electronics cabinets of the MACAO units or the cryocoolers of the other VLT instruments. So far, we have been able to reduce data only for $K=5$ in MR and $K=8.5$ in LR. Within these magnitudes, the accuracy of the AMBER/VLTI transfer function is better than $1 \%$, even with the vibrations, achieved in a few minutes of observations. The improvement of the MACAO system is now achieved.

The HR mode in $K$ has been commissioned down to $K=2$ and is currently offered. The implementation of the FINITO fringe tracker with expected limiting magnitudes of about 7 with the ATs and 10 with the UTs (once the vibration problem is solved) will allow operating in all the spectral resolution modes at this limiting magnitudes. No commissioning of the $J$-band has been made. This commissioning needed the service of the ADC, which has just been successfully tested on sky.

The first results on the differential phase are described by Vannier et al. (2004): they show the RMS instrumental stability of the differential phase over $60 \mathrm{~s}$ in the three spectral bands and in the low resolution mode in the order of $10^{-3}$ rad on two baselines and about $10^{-4} \mathrm{rad}$ on the third one. Some solutions (technical or concerning the data treatment) are under investigation to improve or correct the stability on the two lowest baselines.

The last commissioning with the ATs (July 2006) measured instrumental contrast in the $70 \%-90 \%$ range (including the atmosphere, the VLTI, and AMBER, this contrast being corrected from the source estimated visibility). Similar values should be reached with the UTs when the vibrations are eliminated or actively controlled. The atmosphere alone is supposed to produce about $10 \%$ in contrast loss and the VLTI was initially specified to produce an additional loss lower than $10 \%$. With the currently measured transmission and the atmosphere+VLTI+AMBER fringe contrast, the limiting magnitude can be extrapolated to be between $K=9$ and $K=10$ (Petrov et al. 2007) in the 20\% best conditions. If the vibrations with the UTs are reduced to the level of the ATs and initially specified for the VLTI, these limiting magnitudes will be of the order of $K=11$ with the UTs (Petrov et al. 2007). AMBER is therefore fully compliant with its initial sensibility specifications. 


\section{Conclusion}

To achieve the ambitious astrophysical program of AMBER, it is necessary to obtain visibility measurements with an accuracy better than $10^{-2}$ (goal: $10^{-3}$ ) on sources of magnitude $K$ relatively smaller than 11 and to reach an instrumental stability of the differential phase of $10^{-3} \mathrm{rad}$ (goal: $10^{-4} \mathrm{rad}$ ) over one minute. It was shown that these general specifications are driven by the necessity for the instrument to provide interferometric requirements that consist mainly of contrast level and accuracy, optical throughput and stability. To assess the AMBER performance, a tolerance procedure was defined: with the scientific specifications, the interferometric specifications were determined, in terms of error budgets for the fiber coupling degradation factor due to misalignments and for the instrumental contrast. Both allowed us to define the degrees of freedom of each optical element, respectively located before and after the optical fibers, and represented inputs for the detailed tolerance analysis performed with a ray-tracing tool.

The complete tolerance study, confirmed by the observations, assessed the feasibility of the requirements, i.e., an overall instrumental contrast level higher than $80 \%$ in the $K$-band and the 11th $K$-magnitude with the lowest resolution. After separate integration and tests in the institutes in charge of the warm optics (Robbe-Dubois et al. 2003), SPG (Lisi 2003), and DET, the whole instrument was integrated and tested in LAOG in 2003. AMBER successfully passed the Preliminary Acceptance in Europe in November 2003, resulting in the validation of the instrument laboratory performance, of the compliance with the initial scientific specifications (Rousselet-Perraut et al. 2004), and of the acceptance of ESO for AMBER to be part of the VLTI. After the transportation of the instrument to Paranal, Chile in January 2004, the Assembly Integration and Verification phase occurred mid-March with a successful first fringe observation of bright stars with the VLTI siderostats (Robbe-Dubois et al. 2004).

Since then four commissioning runs have been performed, strengthening the performance. The astrophysical results presented in this issue of A\&A is the best evidence that AMBER is today a very powerful tool allowing unequalled astrophysical knowledge. More results are expected once VLTI has completely solved vibration problems and installed the fringe sensor.

Acknowledgements. The AMBER project ${ }^{1}$ was founded by the French Centre National de la Recherche Scientifique (CNRS), the Max Planck Institute für Radioastronomie (MPIfR) in Bonn, the Osservatorio Astrofisico di Arcetri (OAA) in Firenze, the French Region "Provence Alpes Côte D'Azur" and the European Southern Observatory (ESO). The CNRS funding has been made through the Institut National des Sciences de l'Univers (INSU) and its Programmes Nationaux (ASHRA, PNPS, PNP).

The OAA co-authors acknowledge partial support from MIUR grants to the Arcetri Observatory: A LBT interferometric arm, and analysis of VLTI interferometric data and From Stars to Planets: accretion, disk evolution and planet formation and from INAF grants to the Arcetri Observatory Stellar and Extragalactic Astrophysics with Optical Interferometry. C. Gil work was supported in part by the Fundação para a Ciência e a Tecnologia through project POCTI/CTE-AST/55691/2004 from POCTI, with funds from the European program FEDER.

The preparation and interpretation of AMBER observations benefit from the tools developed by the Jean-Marie Mariotti Center for optical interferometry $\mathrm{JMMC}^{2}$ and from the databases of the Centre de Données Stellaires (CDS) and of the Smithsonian/NASA Astrophysics Data System (ADS).

We would like to thank the successive directors of INSU/CNRS, G. Debouzy, F. Casoli and A.-M. Lagrange, the ASHRA president P. Léna and the ESO VLT

1 The structure and members of the AMBER Consortium can be found in the website: http://amber.obs.ujf-grenoble.fr

2 The JMMC is a center providing software tools for optical interferometry described at the website: http://www.jmmc . fr
Program Manager, M. Tarenghi, for their crucial help in setting up and supporting the Consortium. We also thank S. Bensammar, V. Coudé du Foresto, G. Perrin for their advice in defining the AMBER concept.

We are very grateful to the ESO staff in Garching and Paranal for their help in the design and the commissioning of AMBER.

The key mechanical design and manufacturing have been provided by the Division Technique de l'INSU and by the mechanical workshops of the Observatoire de Bordeaux, Observatoire de la Côte d'Azur and Université de Nice.

\section{Appendix A: Details on the contrast error budget}

\section{A.1. OPD equality}

The fringe contrast degradation factor $\rho_{\text {opd }}$ due to an OPD $\delta$ between the two arms of the interferometer is:

$\rho_{\text {opd }}=\frac{\sin \left(\pi \delta / l_{\mathrm{c}}\right)}{\pi \delta / l_{\mathrm{c}}}$

where $l_{\mathrm{c}}$ is the coherence length equal to $\lambda^{2} / \Delta \lambda$. This is considering a constant spectrum for the source on the observation bandwidth $\Delta \lambda$.

\section{A.2. OPD vibration ( $r m s)$}

Some piston or rms OPD variation effects introduce a complex term in the pupil transmission terms that can be written: $P_{1,2}(x, y)=P_{0}(x, y) . \mathrm{e}^{\mathrm{i} \cdot \phi_{1,2}}$. The associated contrast degradation factor is (Robbe 1996) $\rho_{\text {vib }}=\left\langle\mathrm{e}^{\mathrm{i} . \Delta \phi}\right\rangle$, where $\Delta \phi$ is the instantaneous differential phase and \langle\rangle represents the average during the integration time. From (Roddier 1981):

$\rho_{\mathrm{vib}}=\mathrm{e}^{-\sigma^{2} / 2} \approx 1-\sigma^{2} / 2$,

with $\sigma^{2}$ the phase variance, the approximation being valid for very small variations.

As an example, considering a rms OPD variation of $\lambda / 140$, the phase variance is then $\sigma^{2}=(2 \pi / 140)^{2}$ leading to a degradation factor of 0.999 and a contrast noise $1-\rho_{\text {vib }}$ of $10^{-3}$. Simulations gave the same results and also showed that the degradation is independent of the spectral resolution.

\section{A.3. Static image overlapping errors}

Static tracking errors appear when a differential tilt between the two combined wavefronts exists, inducing a non-perfect overlapping of the two Airy disks. The phase $\phi$ in the wavefront, as a function of a tilt $(\alpha, \beta)$, is given by: $\phi(x, y)=\mathrm{e}^{\mathrm{i} \frac{2 \pi}{\lambda}(\alpha x+\beta y)}$, where $x, y$ are the spatial coordinates and $\alpha, \beta$ are the inclinations of the wavefront. The wavefront becomes:

$\Psi(x, y)=P_{1}(x, y) \mathrm{e}^{\mathrm{i} \frac{2 \pi}{\lambda}\left(\alpha_{1} x+\beta_{1} y\right)}+P_{2}(x, y) \mathrm{e}^{\mathrm{i} \frac{2 \pi}{\lambda}\left(\alpha_{2} x+\beta_{2} y\right)}$ where $P_{1,2}(x, y)=P_{0}(x, y) \otimes \delta\left(x \pm \frac{B}{2}\right)$, with $P_{0}$ the pupil transmission, $\otimes$ the convolution operator, $\delta$ the Dirac's function, and $B$ the beam separation. The associated contrast degradation factor $\rho_{\text {tilt }}$ is (Robbe 1996):

$\rho_{\text {tilt }}=\frac{\widetilde{P}_{0}\left(u-\frac{\alpha_{1}}{\lambda}, v-\frac{\beta_{1}}{\lambda}\right) \widetilde{P}_{0}^{*}\left(u-\frac{\alpha_{2}}{\lambda}, v-\frac{\beta_{2}}{\lambda}\right)}{\left|\widetilde{P}_{0}\left(u-\frac{\alpha_{1}}{\lambda}, v-\frac{\beta_{1}}{\lambda}\right)\right|^{2}+\left|\widetilde{P}_{0}^{*}\left(u-\frac{\alpha_{2}}{\lambda}, v-\frac{\beta_{2}}{\lambda}\right)\right|^{2}}$,

where $\sim$ denotes the Fourier Transform and $*$ the complex conjugate. This term can be analytically expressed considering a coaxial acquisition of the fringes. $\rho_{\text {tilt }}$ can then be approximated by $\rho_{\text {tilt }}=\frac{2 J_{1}(Z)}{Z}$, where $J_{1}$ is the first order Bessel function of the first kind and $Z=\frac{2 \pi R w}{\lambda}$, with $w=\sqrt{\left(\alpha_{1}-\alpha_{2}\right)^{2}+\left(\beta_{1}-\beta_{2}\right)^{2}}$ 
and $R$ the circular pupil radius. For a better estimation, simulations using the AMBER multi-axial configuration were performed to assess the requirements. The requirements are equivalent in any spectral resolution.

\section{A.4. Overlapping jitters (rms)}

Assuming a Gaussian distribution for the pointing errors, the mean contrast was evaluated as a function of the overlapping error standard deviations.

\section{A.5. Wavefront quality ( $r m s)$}

Assuming a Gaussian distribution for the phase defects originated in departures from an ideal shape of the optical surface, the mean contrast is evaluated as a function of the error standard deviations. Simulations show that the results are strongly similar in every spectral bandwidth. The results are quite similar to (a little more optimistic than) the approximation usually used for the degradation factor $\rho_{\mathrm{wfe}}=\mathrm{e}^{-\sigma^{2} / 2}$ where $\sigma$ is the rms differential wavefront error (WFE) in radians.

\section{A.6. Defocus}

The error in focusing was introduced in the simulations via the associated Zernike polynomial. Results show that the contrast loss depends on the spectral bandwidth.

\section{A.7. Polarization: phase delay and differential rotation}

Polarization effects were described by Rousselet-Perraut et al. (1996) and we just give the analytical results here. A contrast degradation can be induced by a differential instrumental polarization. Each direction of polarization, s and p, produces an independent interference pattern. Both add incoherently. A differential phase delay of $\phi_{\mathrm{s}}-\phi_{\mathrm{p}}$ between the interference patterns introduces a fringe shift $\phi^{\prime}=\frac{\phi_{\mathrm{s}}+\phi_{\mathrm{p}}}{2}$ and a visibility degradation factor $\rho_{\mathrm{pol}}$ :

$\rho_{\mathrm{pol}}=\cos \left(\frac{\phi_{\mathrm{s}}-\phi_{\mathrm{p}}}{2}\right)$.

Now, if there exists an angle $\theta_{12}$ between the reference frame between the two arms of the interferometer, the contrast degradation factor $\rho_{\text {rot }}$ is:

$\rho_{\text {rot }}=\frac{2\left|\cos \left(\theta_{12}\right)\right|}{1+\cos ^{2}\left(\theta_{12}\right)}$.

\section{References}

Arsenault, R., Alonso, J., Bonnet, H., et al. 2003, The ESO Messenger, 112, 7

Chelli, A. 2000, AMBER internal memo, AMB-IGR-018

Coudé du Foresto, V. 1997, in Astrofib'96, ed. P. Kern, \& F. Malbet, 27

Escarrat, L. 2000, DEA (Master) Astrophysique, Imagerie et Haute Resolution Angulaire, (University of Nice, Alcatel Space Industies, Observatoire de la Côte d'Azur)

Hofmann, K.-H. 1999, AMBER internal memo, AMB-IGR-013

Lisi, F., Gennari, S., Stefanini, P., et al. 2003, in Cryogenic Optical Systems and Instruments X, ed. J. B. Heaney, \& L. G. Burriesci, SPIE, 5172, 141

Malbet, F. 1999, AMBER internal memo, AMB-IGR-010

Malbet, F., Petrov, R. G., Tallon-Bosc, I., et al. 2003, in Interferometry for Optical Astronomy II, ed. W. A. Traub, SPIE, 4838, 1163
Mège, P., Malbet F., \& Chelli, A. 2000, in Interferometry in Optical Astronomy, ed. P. Lena, \& A. Quirrenbach, SPIE, 4006, 310

Millour, F., Tatulli, E., Chelli, A., et al. 2004, in New Frontiers in Stellar Interferometry, ed. W. A. Traub, SPIE, 5491, 1222

Mourard, D., Bonneau, D., Glentzlin, A., et al. 2000, in Interferometry in Optical Astronomy, ed. P. Lena, \& A. Quirrenbach, SPIE, 4006, 434

Petrov, R. G., Malbet, F., Richichi, A., et al. 2001, C. R. Acad. Sci. Paris, Vol. 2, IV, 67

Petrov, R. G., Vannier, M., Lopez, B., et al. 2003, in EAS Publ. Ser., 8, 297

Petrov, R. G., Malbet, F., Weigelt, G., et al. 2007, A\&A, 464, 1

Puech, F., \& Gitton, Ph. 2005, ESO Document, VLT-ICD-ESO-15000-1826

Richichi, A., Bloecker, T., Foy, R., et al. 2000, in Interferometry in Optical Astronomy, ed. P. Lena, \& A. Quirrenbach, SPIE, 4006, 80

Robbe, S. 1996, PhD dissertation

Robbe-Dubois, S., Bresson, Y., Antonelli, P., et al. 2003, in Interferometry for Optical Astronomy II, ed. W. A. Traub, SPIE, 4838, 1139

Robbe-Dubois, S., Bresson, Y., \& Lagarde, S. 2003, ESO document, VLT-TREAMB-15830-1010

Robbe-Dubois, S., Petrov, R. G., Beckmann, U., et al. 2004, in New Frontiers in Stellar Interferometry, ed. W. A. Traub, SPIE, 5491, 1089

Roddier, F. 1981, Progress in Optics, vol. XIX

Rousselet-Perraut, K., Vakili, F., \& Mourard, D. 1996, Opt. Eng., 35(10), 2943

Rousselet-Perraut, K., LeCoarer, E., Gil, C., et al. 2004, in New Frontiers in Stellar Interferometr, ed. W. A. Traub, SPIE, 5491, 1398

Segransan, D., Beuzit, J.-L., Forveille, T., et al. 2000, in Interferometry in Optical Astronomy, ed. P. Lena, \& A. Quirrenbach, SPIE, 4006, 269

Tallon-Bosc, I. 1999, AMBER internal memo, AMB-IGR-009

Tatulli, E., Mège, P., \& Chelli, A. 2004, A\&A, 418, 1179

Tatulli, E., Millour, F., Chelli, A., et al. 2007, A\&A, 464, 29

Vannier, M. 2003, PhD dissertation

Vannier, M., Petrov, R. G., Robbe, S., et al. 2002, in Scientific Drivers for ESO Future VLT/VLTI Instrumentation, ed. J. Bergeron, \& G. Monnet, (ESO), 340 Vannier, M., Petrov, R. G., Schoeller, M., et al. 2004, in New Frontiers in Stellar Interferometry, ed. W. A. Traub, SPIE, 5491, 577

Wagner, R., \& Tomlinson, W. 1982, Appl. Opt., 21, 2671

1 Laboratoire Universitaire d'Astrophysique de Nice, UMR 6525 Université de Nice - Sophia Antipolis/CNRS, Parc Valrose, 06108 Nice Cedex 2, France e-mail: robbe@unice. fr

2 Laboratoire Gemini, UMR 6203 Observatoire de la Côte d'Azur/CNRS, BP 4229, 06304 Nice Cedex 4, France

3 INAF-Osservatorio Astrofisico di Arcetri, Istituto Nazionale di Astrofisica, Largo E. Fermi 5, 50125 Firenze, Italy

4 Max-Planck-Institut für Radioastronomie, Auf dem Hügel 69, 53121 Bonn, Germany

5 Laboratoire d'Astrophysique de Grenoble, UMR 5571 Université Joseph Fourier/CNRS, BP 53, 38041 Grenoble Cedex 9, France

6 European Southern Observatory, Casilla 19001, Santiago 19, Chile

7 Division Technique INSU/CNRS UPS 855, 1 place Aristide Briand, 92195 Meudon cedex, France

8 ONERA/DOTA, 29 av de la Division Leclerc, BP 72, 92322 Chatillon cedex, France

9 Centre de Recherche Astronomique de Lyon, UMR 5574 Université Claude Bernard/CNRS, 9 avenue Charles André, 69561 Saint Genis Laval Cedex, France

10 IRCOM, UMR 6615 Université de Limoges/CNRS, 123 avenue Albert Thomas, 87060 Limoges Cedex, France

11 European Southern Observatory, Karl Schwarzschild Strasse 2, 85748 Garching, Germany

12 Kiepenheuer Institut für Sonnenphysik, Schöneckstr. 6, 79104 Freiburg, Germany

13 Departamento de Astronomia, Universidad de Chile, Chile

14 Instituut voor Sterrenkunde, KU-Leuven, Celestijnenlaan 200D, 3001 Leuven, Belgium

15 Centro de Astrofísica da Universidade do Porto, Rua das Estrelas, 4150-762 Porto, Portugal

16 Laboratoire Astrophysique de Toulouse, UMR 5572 Université Paul Sabatier/CNRS, BP 826, 65008 Tarbes Cedex, France 